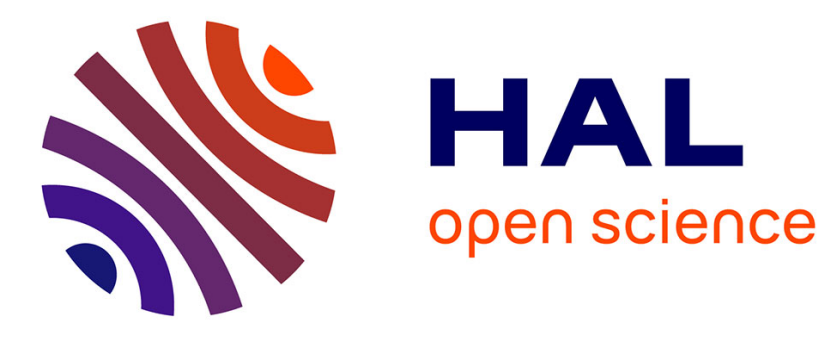

\title{
Definition and diagnosis of constitutional thinness: a systematic review
}

Mélina Bailly, Natacha Germain, Bogdan Galusca, Daniel Courteix, David Thivel, Julien Verney

\section{- To cite this version:}

Mélina Bailly, Natacha Germain, Bogdan Galusca, Daniel Courteix, David Thivel, et al.. Definition and diagnosis of constitutional thinness: a systematic review. British Journal of Nutrition, 2020, 124 (6), pp.531-547. 10.1017/S0007114520001440 . hal-03011966

\section{HAL Id: hal-03011966 https://hal.uca.fr/hal-03011966}

Submitted on 18 Nov 2020

HAL is a multi-disciplinary open access archive for the deposit and dissemination of scientific research documents, whether they are published or not. The documents may come from teaching and research institutions in France or abroad, or from public or private research centers.
L'archive ouverte pluridisciplinaire HAL, est destinée au dépôt et à la diffusion de documents scientifiques de niveau recherche, publiés ou non, émanant des établissements d'enseignement et de recherche français ou étrangers, des laboratoires publics ou privés. 


\title{
Definition and diagnosis of constitutional thinness: a systematic
}

\author{
review
}

\author{
Mélina BAILLY ${ }^{\mathrm{a}, \mathrm{b}}$, Natacha GERMAIN ${ }^{\mathrm{b}, \mathrm{c}}$, Bogdan GALUSCA ${ }^{\mathrm{b}, \mathrm{c}}$, Daniel COURTEIX ${ }^{\mathrm{a}}$, David \\ THIVEL ${ }^{\text {a }}$, Julien VERNEY ${ }^{\mathrm{a}}$
}

a Université Clermont Auvergne, CRNH, AME2P, F-63000 Clermont-Ferrand, France, melina.bailly@uca.fr, julien.verney@uca.fr, david.thivel@uca.fr, daniel.courteix@uca.fr; b Eating Disorders, Addictions and Extreme Bodyweight Research Group (TAPE) EA 7423, Jean Monnet University, Saint-Étienne, France, natacha.germain@chu-st-etienne.fr, bogdan.galusca@chu-stetienne.fr; ${ }^{c}$ Division of Endocrinology, Diabetes, Metabolism and Eating Disorders, CHU SaintÉtienne, France

Corresponding Author: Mélina BAILLY, Université Clermont Auvergne, CRNH, AME2P, F-63000

Clermont-Ferrand, France, melina.bailly@uca.fr

Running head: Diagnosis of constitutional thinness

Keywords: Constitutional thinness; Constitutional leanness; Diagnosis; Weight gain resistance; Underweight

\begin{abstract}
Abbreviations used: AN: anorexia nervosa, BMI: body mass index, BSQ: body shape questionnaire, C: control subjects, CIDI: composite international diagnostic interview, CT: constitutional thinness, DEBQ: dutch eating behavior questionnaire, DSM: diagnostic and statistical manual of mental disorder, EDE: eating disorder examination questionnaire, EDI: eating disorder inventory questionnaire, FFM: fat-free mass, FM: fat mass, FT3: free triiodothyronine, MeSH: medical subject headings, METs: metabolic equivalents, MOSPA: monica optional study of physical activity, NA: not applicable, NR: not reported, PRISMA: preferred reporting items for systematic reviews and meta-analyses, REE: resting energy expenditure, SD: standard deviation, SEM: standard error of the mean, TFEQ: three-factor eating questionnaire, WHO: world health organization
\end{abstract}




\section{Studies enrolling Constitutionally Thin adults}
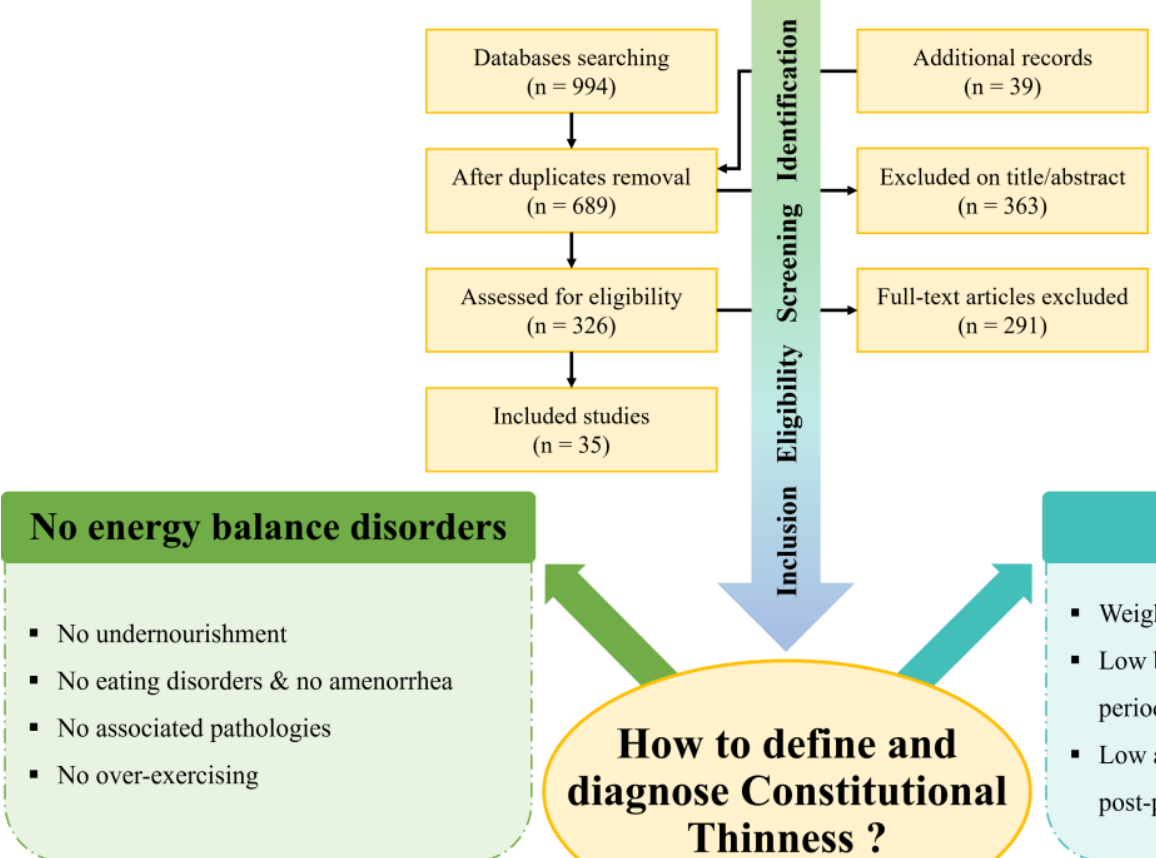

\section{Weight history}

- Weight gain resistance

- Low body mass index during all growth period

How to define and diagnose Constitutional

- Low and stable bodyweight throughout Thinness ?

\section{Need to harmonize our medical and scientific approaches}

- Need to be aware of the existence of Constitutional Thinness

- Need to adopt a common terminology, definition and diagnosis

- Propositions of recommendations

- Development of a decision tree to help in the diagnosis of Constitutional Thinness

\section{Graphical abstract}




\section{Abstract}

The existing literature about the definition and diagnostic criteria of constitutional thinness appears equivocal. The present work systematically reviewed the criteria used in the diagnosis of adult individuals with constitutional thinness (PROSPERO registration number: CRD42019138236). Five electronic bibliographic databases were searched between December 2018 and November 2019: MEDLINE, EMBASE, CENTRAL (Cochrane Library), Google Scholar and CLINICAL TRIALS. Search terms were combined with Medical Subject Headings (MeSH) terms. The search strategy included any clinical trials that enrolled adults with constitutional thinness. Studies were systematically excluded if the state of thinness was not due to a well-identified constitutional origin. From the 689 references after duplicates removal, 199 studies were excluded based on title and 164 based on abstract. According to the inclusion and exclusion criteria, 291 other studies were removed. Finally, 35 studies remained at the end of the process. The analysis of these studies showed high heterogeneity in the diagnostic criteria of constitutional thinness. It emerged the real need to adopt a common terminology and to systematically exclude potential non-constitutional origins of thinness such as eating disorders, associated pathology or over-exercising, with validated tools. Weight history, physiological menses, and weight gain resistance are also important criteria to consider. The present systematic review revealed that our medical and scientific approaches of constitutional thinness need to be harmonized in terms of terminology and diagnostic criteria. Although further studies are needed, we finally proposed recommendations and a decision tree to help in the recognition and diagnosis of constitutional thinness. 


\section{Introduction}

As early as 1933, the existence of constitutional thinness (CT) had already been mentioned by Erich Grafe $^{(1)}$, followed by the first observations of Passmore et al. ${ }^{(2)}$ and Genest et al. ${ }^{(3)}$ in 1955. In a French publication from $1953^{(4)}$, Bernard Wissmer wondered why CT and its treatment had raised so little consideration contrary to obesity. This remark is still valid about sixty years later with obesity and its treatment being widely investigated while CT remains poorly studied ${ }^{(5)}$. Although there is a growing preoccupation for $\mathrm{CT}$ among clinicians due to an increasing number of individuals presenting thinness and seeking to gain weight without apparent criteria of anorexia nervosa (AN); the prevalence of CT remains difficult to determine ${ }^{(5)}$, but would be less than $0.4 \%$ for males and less than $2.7 \%$ for females (underweight from all causes) ${ }^{(6)}$. Despite a large proportion of concerned individuals, a lot of them do not consult because of a lack of recognition and diagnosis of this condition. Given this lack of interest in literature, CT is poorly described, which can favor its misunderstanding and misdiagnosis ${ }^{(5)}$, mainly with AN. Although CT and AN are both characterized by a low body mass index (BMI), people with CT do not present eating disorders, food restriction, psychological disorders, hormonal signs of undernutrition but present an equilibrated energy metabolism, stable bodyweight within lower percentiles of growth curve and physiological menses for females ${ }^{(7-11)}$. Despite these clinical differences, the distinction between AN and CT remains difficult. Guy-Grand \& Badevant proposed a first decision tree to diagnose $\mathrm{CT}$ in the early eighties ${ }^{(12)}$, but its diagnosis is still debated; especially with the removal of amenorrhea criterion from the definition of AN in the Diagnostic and Statistical Manual of Mental Disorder V (DSM-V) ${ }^{(8,13)}$. In our modern societies, individuals with CT have to face social stigmatization similar to that of anorectic patients ${ }^{(14)}$, due to their low bodyweight and corpulence. Unlike patients with AN, people with CT show an important desire to gain weight, which is the main reason for medical consultation ${ }^{(5)}$. As already noted in $1982^{(15)}$, the demand of individuals with CT for clinical examination is stereotyped; they are concerned about their thinness and dissatisfied with their morphology usually judged for its lack of femininity for women or virility for men. CT seems then to be a natural state of underweight leading to a high self-dissatisfaction and whose causes remain unclear. While absolute resting energy expenditure (REE) was found lower ${ }^{(7,8,10)}$ or similar ${ }^{(16-18)}$ in CT 
individuals vs normal-weight control subjects, REE-to-fat-free mass (FFM) ratio was found higher in CT vs control subjects in some studies ${ }^{(7,18)}$, but not significantly higher in some other studies ${ }^{(10,17,19)}$. Other evidence seems to indicate a more pronounced brown fat activity in $\mathrm{CT}^{(20)}$. Despite an apparently similar energy intake (quantitatively as well as qualitatively) as normal-weight people ${ }^{(5,7,9,10,19)}$, specific physiological control of appetite has been suggested in individuals with CT ${ }^{(9-11,21-23)}$, with for instance an earlier and higher satiety onset during meals leading to reduced but more frequent intakes (more inbetween meal snacking) ${ }^{(10)}$. CT subjects present no eating disorder-related traits and even have lower food restrictive behaviors compared to normal-weight people ${ }^{(8,10)}$. Despite their low BMI, they present a non-blunted fat mass $(\mathrm{FM})$ percentage ${ }^{(7,8,10,11,17,19,23-27)}$. However, CT people display impairments in their bone quality: small bone sizes, low bone mass, low calculated breaking strength ${ }^{(28)}$, low bone mineral density ${ }^{(19,24,26,28)}$, but however apparent normal bone turnover ${ }^{(28)}$. Even if the potential increased risk of osteoporosis with aging in CT remains to be robustly demonstrated, these bone impairments could be considered as the main comorbidity associated with CT. This public health concern might not be the only one, but issues in the recognition and diagnosis of CT likely lead to a lack of knowledge. With 2.5 thin subjects per family in CT $v s 0.5$ in AN, CT is strongly suggested to be a heritable trait likely attributable to genetic factors ${ }^{(7,29,30)}$. Moreover, the exploration of the genetic architecture of thinness demonstrated the polygenic component of CT: genome-wide association studies revealed evidence of loci that could confer susceptibility of $\mathrm{CT}$ and also be informative in the identification of potential anti-obesity targets ${ }^{(30)}$. While there is a growing scientific and clinical interest to better understand and characterize $\mathrm{CT}$, the used inclusion and exclusion criteria remain highly heterogeneous in-between studies, making any comparison and conclusion difficult. This high variability in CT diagnosis underlines today a clear need for a common definition of CT and harmonized criteria that should be used for CT detection. According to the recent literature ${ }^{(8,10,18,23,30,31)}$, parameters such as the terminology used, the characterization and fluctuation of the level of thinness, the consideration of psychological or physical illnesses, the weight gain resistance, or the level of physical activity appear, a priori, to be the main parameters to focus on in this systematic review. Thus, the present paper 
proposed a systematic analysis of all the parameters used so far as inclusion criteria of CT individuals in the available studies, trying to suggest a clear definition and diagnostic method of CT.

\section{Materials and Methods}

The systematic literature search was performed following the Preferred Reporting Items for Systematic Reviews and Meta-Analyses (PRISMA) guidelines and was registered in the International Prospective Register of Systematic Reviews (PROSPERO registration number: CRD42019138236).

\section{Search strategy}

The search was conducted on CT and aimed to include any clinical trials enrolling a group of adults with CT. Five electronic bibliographic databases were searched between December 2018 and November 2019: MEDLINE, EMBASE, CENTRAL (Cochrane Library), GOOGLE SCHOLAR and CLINICAL TRIALS. Relevant keywords were discussed and selected between the co-authors. Search terms were also combined with MeSH terms. The following syntax was finally used to search on the MEDLINE database: ((constitution[TI] OR constitutional[TI] OR constitutionally[TI]) AND (thinness[TI] OR leanness[TI] OR thin[TI] OR lean[TI])) OR "constitutional thinness"[TW] OR "constitutional leanness"[TW] OR (((resistance[TI] OR resistant[TI]) AND "weight gain"[TI]) NOT "insulin resistance"[TI]) OR ("thinness/physiology"[Mesh] OR ((physiological[TI] OR physiologically[TI] OR physiology[TI]) AND (thinness[TI] OR leanness[TI] OR thin[TI] OR lean[TI])) NOT "obesity"[Mesh]) AND ("humans"[Mesh] OR "humans"[TW] OR "human"[TW]). Searches were carried out on articles published from 1950. Adapted syntaxes were used to perform the search on the other databases. The authors collectively discussed any discrepancies. All the selected references were then extracted to Zotero Software (5.0.21, CHNM, GMU, USA). 


\section{Study eligibility}

\section{Inclusion criteria}

Clinical trials had to be published in English or French languages and had to enroll constitutional thin/lean adult females or males. Any fields of study could be included in the analysis. However, experiments on animals and clinical trials on children were not eligible for the systematic review. In addition, studies were not included if not enough data were available: letters to the Editor, reviews, abstracts alone, or case studies. Only thinness due to a "constitutional" origin was considered. To do this, papers had to mention at least one of these criteria: "constitutional thin/lean" keywords, state of thinness confirmed by measurements, absence of eating disorders, no over-exercising, no associated pathology, physiological menstruations, stable bodyweight, and/or weight gain resistance/desire.

\section{Exclusion criteria}

Studies were excluded if thinness was not due to a well-identified constitutional origin, such as associated diseases, undernourishment, eating disorders, over-exercising, or any "non-constitutional" origins causing a state of thinness. Specific attention was given to the large number of studies that wrongly named their normal-weight control groups as "lean" groups. Normal-weight "lean" control groups were not considered as "constitutional lean" groups and were therefore excluded from the systematic review.

\section{Data extraction and synthesis of results}

After the removal of duplicates, a first selection was performed on titles and abstracts of studies to assess eligibility of identified records through databases searching. Full-text articles were then screened and included according to the aforementioned inclusion and exclusion criteria. At each step of this process, a second screener assessed independently the identification, eligibility, and inclusion of papers. Any disagreements about the eligibility and inclusion of papers or about the appraisal of methodological 
quality were solved by discussing with a third reviewer until a consensus was reached. Potentially relevant references cited in full-text read articles were also added to the initial search. Computer files containing the selected papers at each stage of the selection process were developed and made available to all the co-authors. At the end of the process, 35 studies were collectively included in the analysis. The flow diagram of identification, screening, eligibility and inclusion process is provided in Figure 1. Data extraction of the 35 selected papers was performed using a standardized extraction spreadsheet to collect relevant information. As presented in Table 1, relevant information was summarized on established parameters chosen collectively by the authors: reference, population characteristics, definition of thinness, consideration of the absence of eating disorders, consideration of other main parameters, and areas of study. We mean by "presence of terminology" (Table 1) the explicit mention of "constitutional(ly) thin(ness)/lean(ness)" keywords. Outcome variables were not assessed in the present work: only the inclusion criteria of the selected studies were considered. Parameters such as food questionnaires or nutritional markers do not appear in Table 1 if these parameters were used as outcomes after the constitution of groups and not as inclusion criteria. Studies were listed in Table 1 according to the publication year, from the oldest to the most recent. Since this systematic review focuses on diagnostic criteria, it was not considered appropriate to retain studies from the same cohorts (recorded as duplicates).

\section{Risks of bias}

The Cochrane Collaboration's tool ${ }^{(32)}$ was used to assess the risks of bias; as presented in Table 2. Two authors estimated independently the risks of bias in each included study. The following criteria were assessed: random sequence generation (selection bias), allocation concealment (selection bias), blinding of participants and personnel (performance bias), blinding of outcome assessment (detection bias), incomplete outcome data (attrition bias) and selective reporting (reporting bias). Any disagreements were discussed with a third co-author until a consensus was reached. No study was excluded based on the risks of bias. 


\section{Results}

The initial database search yielded a total of 994 studies and 39 additional studies were also identified.

In total, 689 studies remained after the removal of duplicates. After the review of titles and abstracts, 363 studies were excluded: 199 based on title and 164 based on abstract. Thus, 326 full-text articles were scrutinized for eligibility according to inclusion and exclusion criteria. Finally, 35 studies were considered for analysis (Figure 1). The risks of bias were estimated with the Cochrane Collaboration's tool $^{(32)}$ as presented in Table 2.

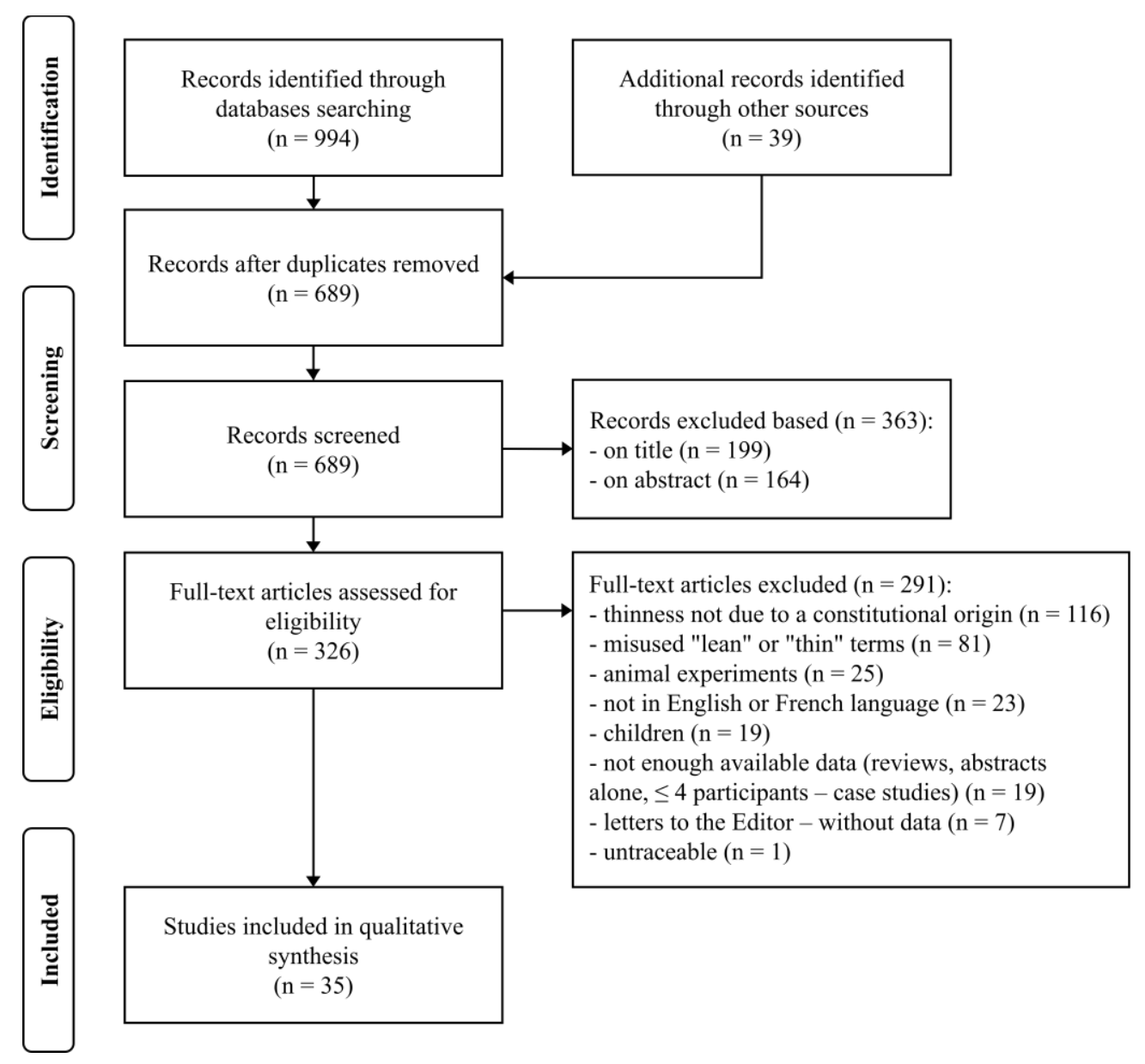

Figure 1. Flow diagram of the description of the screening, selection and inclusion process 


\section{Population characteristics}

Of the 35 studies selected in the systematic review, $26^{(7-11,16,17,19-28,33-41)}$ enrolled females exclusively, 3 ${ }^{(18,42,43)}$ enrolled males exclusively and $6^{(30,31,44-47)}$ enrolled both females and males (Table 1). Of these 35 studies, 32 (7-11,16-28,30,31,33-37,39-42,45-47) included a normal-weight control group and $23^{(7-9,11,16-18,20-}$ $25,27,28,30,34,35,37,38,41,44,46)$ included a group of individuals with AN (18 ${ }^{(7-9,11,16-18,20-24,27,28,35,37,41,46)}$ of restrictive type, $2^{(38,44)}$ of both restrictive and binge eating/purging type and $3^{(25,30,34)}$ did not report the type of AN). Selected studies included sample sizes ranging from $6^{(37)}$ to $1622^{(30)}$ (both genders) in individuals with CT, from $7^{(7,9)}$ to $10433^{(30)}$ (both genders) in normal-weight control people and from $6^{(7)}$ to $96^{(44)}$ (both genders) in patients with AN. Studies enrolled participants from $19.4^{(25)}$ to $42.4^{(36)}$ years old in people with CT, from $19.3^{(24)}$ to $52.3^{(30)}$ years old (both genders) in normal-weight people and from $15.3^{(44)}$ to $26.4^{(41)}$ years old in patients with AN. BMI ranged from $15.7^{(9,11)}$ to $22.5^{(42)} \mathrm{kg} . \mathrm{m}^{-}$ 2 in individuals with CT, from $20.3^{(37)}$ to $27.6^{(42)} \mathrm{kg} \cdot \mathrm{m}^{-2}$ in normal-weight controls and from $12.0^{(38)}$ to $17.1^{(18)} \mathrm{kg} \cdot \mathrm{m}^{-2}$ in patients with AN.

\section{Definition of thinness}

The "constitutional(ly) thin(ness)/lean(ness)" keywords were mentioned in $28^{\text {(7-11,17-23,25-28,30,31,33,35-38,41- }}$ ${ }^{43,46,47)}$ of the 35 studies and were therefore not mentioned in the 7 remaining studies ${ }^{(16,24,34,39,40,44,45)}$. Of the 35 included studies, thinness threshold was reported through absolute BMI value in 21 studies ${ }^{(7-}$ 10,16,19,21-28,30,31,35,37,41,45,47) (ranging from $16.5^{(7,9,21-23,28)}$ to $20.0 \mathrm{~kg} \cdot \mathrm{m}^{-2}(35)$ ), through BMI percentile in one study ( $\leq 15$ th BMI percentile) ${ }^{(44)}$, through percentage of ideal bodyweight in 2 studies (at least $25 \%$ lower than the average ideal bodyweight ${ }^{(33)}$ or $80-90 \%$ of ideal bodyweight ${ }^{(34)}$ ), through silhouette ratings (1: very thin, 9: very large) in 2 studies (ranging from 1 to 3 for thin females ${ }^{(36)}$ and from 1 to 4 for thin males ${ }^{(42)}$ ), through FM percentage in one study ${ }^{(43)}$ (body fat $\leq 20 \%$ and low or normal weight), and through both BMI $\left(<20 \mathrm{~kg} \cdot \mathrm{m}^{-2}\right)$ and FM percentage (between 10 and $\left.20 \%\right)$ in one study ${ }^{(40)}$. Thinness threshold was not clearly reported in $7^{(11,17,18,20,38,39,46)}$ of the 35 studies. Weight history was considered in $25^{(7-10,16-19,21-23,27,28,30,31,35,36,38-40,42,44-47)}$ of the 35 studies: 4 studies (16,39,40,45) reported a stable 
bodyweight for a certain period of time before the experiment (ranging from one week ${ }^{(45)}$ to 2 years $\left.{ }^{(16,39,40)}\right)$ and 21 studies ${ }^{(7-10,17-19,21-23,27,28,30,31,35,36,38,42,44,46,47)}$ reported it for a longer period throughout the growth period and/or the post-pubertal period. Weight history was not considered in the $10^{(11,20,24-}$ $26,33,34,37,41,43)$ remaining studies.

\section{Consideration of the absence of eating disorders in individuals with CT}

Of the 35 studies, $32^{(7-11,16-28,30,31,33-38,41-46)}$ considered the absence of eating disorders in the inclusion criteria of CT and $3^{(39,40,47)}$ did not consider it. The absence of eating disorders was implicitly confirmed by the presence of a group of patients with AN in 23 studies $(7-9,11,16-18,20-25,27,28,30,34,35,37,38,41,44,46)$. This absence of eating disorders was confirmed using questionnaires in 5 studies ${ }^{(10,30,31,35,41)}$, interviews in 3 studies ${ }^{(36,42,46)}$, and both in one study ${ }^{(44)}$. Different questionnaires and thresholds were used: the threefactor eating questionnaire (TFEQ) ${ }^{(48)}$ for 2 studies ${ }^{(41,44)}$ with a cognitive restraint score $\leq 5^{(44)}$ or $\geq 13$ ${ }^{(41)}$ using their respective version of the TFEQ, a food questionnaire with normal scores not further defined for one study ${ }^{(35)}$, the dutch eating behavior questionnaire (DEBQ) ${ }^{(49)}$ and the eating disorder examination questionnaire (EDE) ${ }^{(50)}$ without reported thresholds for 2 studies ${ }^{(10,31)}$, the eating disorder inventory questionnaire (EDI) ${ }^{(51)}$ and the body shape questionnaire (BSQ) ${ }^{(52)}$ without reported thresholds for one study ${ }^{(31)}$, and the SCOFF questionnaire ${ }^{(53)}$ without reported thresholds for one study ${ }^{(30)}$. The Composite International Diagnostic Interview (CIDI) ${ }^{(54)}$ was used for one study ${ }^{(44)}$, the Structured Clinical Interview for DSM-III-R was used for 2 studies ${ }^{(36,42)}$, and an interview to detect potential lifetime eating disorders in accordance with the criteria of the DSM-IV was used for one study (46). The 26 remaining studies ${ }^{(7-9,11,16-28,33,34,37-40,43,45,47)}$ did not mentioned the use of questionnaires or interviews. Three studies ${ }^{(10,19,31)}$ presented the following criteria as inclusion criteria: normal insulinlike growth factor-1 (IGF-1), estradiol and free triiodothyronine $\left(\mathrm{FT}_{3}\right)$. Among them, 2 studies ${ }^{(10,31)}$ also added normal mean cortisol and non-blunted leptin as inclusion criteria. Under nutritional markers were not assessed in the 32 remaining studies ${ }^{(7-9,11,16-18,20-28,30,33-47)}$. 


\section{Consideration of other important parameters in individuals with CT}

Of the 35 studies, $26^{(7-11,16,17,20-28,31,34,35,37-41,46,47)}$ mentioned the presence of menses in their group of CT, $6^{(19,30,33,36,44,45)}$ did not mention it and 3 studies ${ }^{(18,42,43)}$ did not enroll females but only males (not applicable criterion). Weight gain resistance/desire was taken into consideration in 14 articles ${ }^{(7-10,16,21-}$ 23,27,28,31,43,46,47) and was not reported in the 21 other selected studies ${ }^{(11,17-20,24-26,30,33-42,44,45)}$. Among them, 12 studies ${ }^{(7-10,21-23,27,28,31,46,47)}$ specifically referred to the idea of a "desire" to gain weight, one study ${ }^{(16)}$ reported a complaint about being chronically underweight, and one study ${ }^{(43)}$ identified a difficulty in gaining weight. No studies used the term "resistance" to weight gain. The absence of associated pathology was considered in $28^{(7-10,16-23,25-28,30,31,33,34,37,39-41,43,44,46,47)}$ of the 35 studies but was not reported in the 7 remaining studies $(11,24,35,36,38,42,45)$. Physical activity was reported in 13 studies $(7,10,16,17,22,23,25,28,30,31,39,40,47)$ and was consequently not reported in the 22 remaining studies $(8,9,11,18-$ 21,24,26,27,33-38,41-46). Ten articles $(7,16,17,22,25,28,30,39,40,47)$ just mentioned the absence of over-exercising without questionnaire-based assessment. Among them, 2 articles ${ }^{(39,40)}$ specified that participants did not spend more than one hour per week on sport activities and one article ${ }^{(30)}$ excluded all participants who stated that they exercised more than 3 times a week or with an intensity exceeding 6 metabolic equivalents (METs) for any duration or frequency ${ }^{(55)}$. Three articles ${ }^{(10,23,31)}$ used the Monica Optional Study of Physical Activity (MOSPA) questionnaire ${ }^{(56)}$ to assess the absence of over-exercising and one ${ }^{(31)}$ of them added intensive physical activity (more than 3 sessions of physical activity per week) as an exclusion criterion.

\section{Areas of study}

Various fields of study were investigated in the selected articles. Of the 35 studies included in the systematic review, $21^{(7-11,17-28,31,37,43,45)}$ investigated body composition, $19^{(7-11,19,21-24,26-28,31,34,35,37,38,40)}$ assessed hormonal or biochemical parameters and $15^{(7-10,16-20,26,31,35,40,43,45)}$ studied energy balance of individuals with CT. Investigations were carried out in a total of 8 studies ${ }^{(9-11,21-23,31,38)}$ on appetiteregulating hormones, 6 studies ${ }^{(8,19,24,28,31,45)}$ on bone tissue or bone markers, 7 studies ${ }^{(7,8,10,31,36,41,42)}$ on 
psychological profile, 5 studies ${ }^{(10,19,30,31,44)}$ on genetics or omics approaches, 4 studies ${ }^{(19,31,39,40)}$ on muscle tissue or muscle function, 2 studies ${ }^{(16,20)}$ on thermogenesis or brown adipose tissue, one study (47) on ophthalmology, one study ${ }^{(33)}$ on pregnancy, one study ${ }^{(35)}$ on cardiology, one study ${ }^{(46)}$ on functional dyspepsia and one study ${ }^{(41)}$ on neurology. 
Table 1. Inclusion criteria used for diagnosis of constitutional thinness in the clinical trials selected in the systematic review

\begin{tabular}{|c|c|c|c|c|c|}
\hline Reference & $\begin{array}{l}\text { Population characteristics } \\
\text { (sample size, age; BMI) (means } \pm \\
\text { SD) }\end{array}$ & Definition of thinness & $\begin{array}{l}\text { Consideration of the absence of eating } \\
\text { disorders in individuals with CT }\end{array}$ & $\begin{array}{l}\text { Consideration of other main } \\
\text { parameters in individuals with CT }\end{array}$ & $\begin{array}{l}\text { Areas } \\
\text { of } \\
\text { study }\end{array}$ \\
\hline $\begin{array}{l}\text { Schneider et } \\
\text { al., } 1979^{(33)}\end{array}$ & $\begin{array}{l}\text { Females: } \\
\text { CT: } \mathrm{n}=53 ; 25.3 \pm 5.2^{\S} \mathrm{yr} ; \mathrm{NR} \\
\text { C: } \mathrm{n}=100 ; 25.8 \pm 4.2^{\S} \mathrm{yr} ; \mathrm{NR}\end{array}$ & $\begin{array}{l}\text { Presence of terminology } \\
\text { Thinness threshold: at least } 25 \% \text { lower } \\
\text { than the average ideal weight defined for } \\
\text { the height at the first prenatal consultation } \\
\text { (first trimester of pregnancy) } \\
\text { No apparent consideration of weight } \\
\text { history }\end{array}$ & $\begin{array}{l}\text { Considered } \\
\text { No group of AN } \\
\text { Not explicitly confirmed by } \\
\text { questionnaire or interview } \\
\text { Under nutritional markers: NR }\end{array}$ & $\begin{array}{l}\text { Amenorrhea: NR } \\
\text { Weight gain resistance/desire: NR } \\
\text { Healthy, absence of associated } \\
\text { pathology } \\
\text { Physical activity: NR }\end{array}$ & 9 \\
\hline $\begin{array}{l}\text { van } \\
\text { Binsbergen } \\
\text { et al, } 1990 \\
\text { (34) }\end{array}$ & $\begin{array}{l}\text { Females: } \\
\text { CT: } \mathrm{n}=10 ; 26.4^{\S} \mathrm{yr} ; 18.4^{\S} \mathrm{kg} \cdot \mathrm{m}^{-2} \\
\mathrm{C}: \mathrm{n}=10 ; 25.1^{\S} \mathrm{yr} ; 20.8^{\S} \mathrm{kg} \cdot \mathrm{m}^{-2} \\
\text { AN: } \mathrm{n}=20 ; 24.8^{\S} \mathrm{yr} ; 14.3^{\S} \mathrm{kg} \cdot \mathrm{m}^{-2} \\
\text { (AN type: NR) }\end{array}$ & $\begin{array}{l}\text { Absence of terminology } \\
\text { Thinness threshold: } 80-90 \% \text { of ideal } \\
\text { bodyweight } \\
\text { No apparent consideration of weight } \\
\text { history }\end{array}$ & $\begin{array}{l}\text { Considered } \\
\text { Implicitly confirmed by the presence of } \\
\text { a group of AN (DSM-III) } \\
\text { Not explicitly confirmed by } \\
\text { questionnaire or interview } \\
\text { Under nutritional markers: NR }\end{array}$ & $\begin{array}{l}\text { No amenorrhea } \\
\text { Weight gain resistance/desire: NR } \\
\text { Healthy, absence of associated } \\
\text { pathology } \\
\text { Physical activity: NR }\end{array}$ & 3 \\
\hline $\begin{array}{l}\text { Diaz et al., } \\
1992^{(43)}\end{array}$ & $\begin{array}{l}\text { Males: } \\
\text { CT: } n=7 ; 26.3 \pm 4.5 \text { yr; } 21.7 \pm 1.3 \\
\text { kg.m }{ }^{-2}\end{array}$ & $\begin{array}{l}\text { Presence of terminology } \\
\text { Thinness threshold: body fat } \leq 20 \% \text { (and } \\
\text { low or normal weight) } \\
\text { No apparent consideration of weight } \\
\text { history }\end{array}$ & $\begin{array}{l}\text { Considered } \\
\text { No group of AN } \\
\text { Not explicitly confirmed by } \\
\text { questionnaire or interview } \\
\text { Under nutritional markers: NR }\end{array}$ & $\begin{array}{l}\text { Criterion of amenorrhea: NA (males) } \\
\text { Weight gain resistance/desire: } \\
\text { mentioned (they declared themselves to } \\
\text { be good eaters and claimed to have } \\
\text { difficulty gaining weight) } \\
\text { Healthy, absence of associated } \\
\text { pathology } \\
\text { Physical activity: NR }\end{array}$ & 1,2 \\
\hline $\begin{array}{l}\text { Scalfi et al., } \\
1992^{(16)}\end{array}$ & $\begin{array}{l}\text { Females: } \\
\text { CT: } n=7 ; 28.6 \pm 5.6 \mathrm{yr} ; 16.8 \pm 0.8 \\
\text { kg.m }{ }^{-2} \\
\text { C: } n=8 ; 28.5 \pm 3.4 \mathrm{yr} ; 22.5 \pm 2.5 \\
\text { kg.m }{ }^{-2} \\
\text { AN: } n=7 ; 21.3 \pm 3.7 \mathrm{yr} ; 15.3 \pm 2.1 \\
\text { kg.m }{ }^{-2} \\
\text { (AN: restrictive-type) }\end{array}$ & $\begin{array}{l}\text { Absence of terminology } \\
\text { Thinness threshold: BMI }<18.5 \mathrm{~kg} . \mathrm{m}^{-2} \\
\text { Consideration of personal weight history } \\
\text { (stable in the } 2 \text { years before the experiment } \\
\pm 1.5 \mathrm{~kg} \text { by interview) }\end{array}$ & $\begin{array}{l}\text { Considered } \\
\text { Implicitly confirmed by the presence of } \\
\text { a group of AN (DSM-III) } \\
\text { Not explicitly confirmed by } \\
\text { questionnaire or interview } \\
\text { Under nutritional markers: NR (but no } \\
\text { clinical or biochemical evidence of } \\
\text { hyperthyroidism) }\end{array}$ & $\begin{array}{l}\text { No amenorrhea } \\
\text { Weight gain resistance/desire: } \\
\text { mentioned (they complained of being } \\
\text { chronically underweight and perceived } \\
\text { themselves as normal eaters or large } \\
\text { eaters) } \\
\text { Healthy, absence of associated } \\
\text { pathology } \\
\text { Absence of over-exercising }\end{array}$ & 1,10 \\
\hline $\begin{array}{l}\text { Hinney et al., } \\
1997^{(44)}\end{array}$ & $\begin{array}{l}\text { Females: } \\
\text { CT: } n=48 ; 24.7 \pm 3.9 \mathrm{yr} ; 17.6 \pm 0.8 \\
\text { kg.m }{ }^{-2} \\
\text { AN: } n=92 ; 16.6 \pm 3.4 \mathrm{yr} ; 14.5 \pm \\
1.5 \mathrm{~kg} . \mathrm{m}^{-2}\end{array}$ & $\begin{array}{l}\text { Absence of terminology } \\
\text { Thinness threshold: } \leq 15 \text { th BMI percentile } \\
\text { Consideration of personal weight history } \\
\text { (semi-structured interview to assess weight }\end{array}$ & $\begin{array}{l}\text { Considered (DSM-IV) } \\
\text { Implicitly confirmed by the presence of } \\
\text { a group of AN (DSM-IV) } \\
\text { Confirmed by questionnaire and } \\
\text { interview (TFEQ with a cognitive }\end{array}$ & $\begin{array}{l}\text { Amenorrhea: NR } \\
\text { Weight gain resistance/desire: NR } \\
\text { Healthy, absence of associated } \\
\text { pathology } \\
\text { Physical activity: NR }\end{array}$ & 7 \\
\hline
\end{tabular}




\begin{tabular}{|c|c|c|c|c|c|}
\hline & $\begin{array}{l}\text { (AN: restrictive and binge } \\
\text { eating/purging type) } \\
\text { Males: } \\
\text { CT: } n=64 ; 26.1 \pm 4.1 \mathrm{yr} ; 19.0 \pm 1.0 \\
\mathrm{~kg} \cdot \mathrm{m}^{-2} \\
\text { AN: } \mathrm{n}=4 ; 15.3 \pm 0.9 \mathrm{yr} ; 13.9 \pm 2.0 \\
\mathrm{~kg} \cdot \mathrm{m}^{-2} \\
\text { (AN: restrictive and binge } \\
\text { eating/purging type) }\end{array}$ & $\begin{array}{l}\text { history up to age } 18 \text { - at ages } 10,15 \text { and } \\
\text { 18) }\end{array}$ & $\begin{array}{l}\text { restraint score } \leq 5 \text { and CIDI in } \\
\text { accordance with DSM-IV) } \\
\text { Under nutritional markers: NR }\end{array}$ & & \\
\hline $\begin{array}{l}\text { Petretta et al., } \\
1997^{(35)}\end{array}$ & $\begin{array}{l}\text { Females: } \\
\text { CT: } n=10 ; 22 \pm 3 \text { yr; } 16.6 \pm 1.1 \\
\text { kg.m }{ }^{-2} \\
\text { C: } n=10 ; 21 \pm 3 \text { yr; } 23.4 \pm 2.4 \\
\text { kg.m }{ }^{-2} \\
\text { AN: } n=13 ; 20 \pm 2 \text { yr; } 15.7 \pm 2.4 \\
\text { kg.m }{ }^{-2} \\
\text { (AN: restrictive-type) }\end{array}$ & $\begin{array}{l}\text { Presence of terminology } \\
\text { Thinness threshold: BMI }<20 \mathrm{~kg} . \mathrm{m}^{-2} \\
\text { Consideration of personal weight history } \\
\text { (history of leanness throughout life) }\end{array}$ & $\begin{array}{l}\text { Considered } \\
\text { Implicitly confirmed by the presence of } \\
\text { a group of AN (DSM-IV) } \\
\text { Confirmed by questionnaire (normal } \\
\text { scores on food questionnaire - not } \\
\text { further defined) } \\
\text { Under nutritional markers: NR }\end{array}$ & $\begin{array}{l}\text { No amenorrhea } \\
\text { Weight gain resistance/desire: NR } \\
\text { Healthy, associated pathology: NR } \\
\text { Physical activity: NR }\end{array}$ & $\begin{array}{l}1,3 \\
12\end{array}$ \\
\hline $\begin{array}{l}\text { Slof et al., } \\
2003^{(36)}\end{array}$ & $\begin{array}{l}\text { Females: } \\
\text { CT: } n=80 ; 42.4 \pm 7.2 \mathrm{yr} ; 20.3 \pm 1.5 \\
\mathrm{~kg} \cdot \mathrm{m}^{-2} \\
\text { C: } \mathrm{n}=881 ; 43.0 \pm 7.7 \mathrm{yr} ; 26.8 \pm 6.2 \\
\mathrm{~kg} \cdot \mathrm{m}^{-2}\end{array}$ & $\begin{array}{l}\text { Presence of terminology (but "persistent } \\
\text { thinness" preferentially used) } \\
\text { Thinness threshold: } 1-3 \text { (1: very thin, } 9 \\
\text { very large) on silhouette ratings } \\
\text { Consideration of personal weight history } \\
\text { (persistent thinness with consideration of } \\
\text { childhood, adolescence and adulthood) }\end{array}$ & $\begin{array}{l}\text { Considered (DSM-III-R and DSM-IV) } \\
\text { No group of AN } \\
\text { Confirmed by interview (Structured } \\
\text { Clinical Interview for DSM-III-R by } \\
\text { trained interviewers - } 40 \text { hours of } \\
\text { training) } \\
\text { Under nutritional markers: NR } \\
\end{array}$ & $\begin{array}{l}\text { Amenorrhea: NR } \\
\text { Weight gain resistance/desire: NR } \\
\text { Healthy, associated pathology: NR } \\
\text { Physical activity: NR }\end{array}$ & 11 \\
\hline $\begin{array}{l}\text { Tolle et al., } \\
2003^{(11)}\end{array}$ & $\begin{array}{l}\text { Females: } \\
\text { CT: } \mathrm{n}=8 ; 23.3 \pm 3.1^{\S} \mathrm{yr} ; 15.7 \pm \\
0.4^{\S} \mathrm{kg} \cdot \mathrm{m}^{-2} \\
\mathrm{C}: \mathrm{n}=10 ; 23.2 \pm 1.1^{\S} \mathrm{yr} ; 21.5 \pm \\
0.7^{\S} \mathrm{kg} \cdot \mathrm{m}^{-2} \\
\text { AN: } \mathrm{n}=9 ; 17.2 \pm 0.9^{\S} \mathrm{yr} ; 14.6 \pm \\
0.4^{\S} \mathrm{kg} \cdot \mathrm{m}^{-2} \\
\text { (AN: restrictive-type) }\end{array}$ & $\begin{array}{l}\text { Presence of terminology } \\
\text { Thinness threshold: NR (but BMI similar } \\
\text { to the AN group before renutrition) } \\
\text { No apparent consideration of weight } \\
\text { history }\end{array}$ & $\begin{array}{l}\text { Considered } \\
\text { Implicitly confirmed by the presence of } \\
\text { a group of AN (DSM-IV) } \\
\text { Not explicitly confirmed by } \\
\text { questionnaire or interview } \\
\text { Under nutritional markers: NR }\end{array}$ & $\begin{array}{l}\text { No amenorrhea } \\
\text { Weight gain resistance/desire: NR } \\
\text { Healthy, associated pathology: NR } \\
\text { Physical activity: NR }\end{array}$ & $2,3,4$ \\
\hline $\begin{array}{l}\text { Bosy- } \\
\text { Westphal et } \\
\text { al., } 2004^{(45)}\end{array}$ & $\begin{array}{l}\text { CT (12 females): } \mathrm{n}=12 ; 26.4 \pm 6.8 \\
\text { yr; } 16.9 \pm 0.9 \mathrm{~kg} \cdot \mathrm{m}^{-2} \\
\mathrm{C}(12 \text { females and } 13 \text { males }): \\
\mathrm{n}=25 ; 25.4 \pm 2.4 \mathrm{yr} ; 22.3 \pm 2.0 \\
\mathrm{~kg} \cdot \mathrm{m}^{-2}\end{array}$ & $\begin{array}{l}\text { Absence of terminology } \\
\text { Thinness threshold: BMI }<18.5 \mathrm{~kg} \cdot \mathrm{m}^{-2} \\
\text { Consideration of personal weight history } \\
\text { (stable for at least one week) }\end{array}$ & $\begin{array}{l}\text { Considered (DSM-IV) } \\
\text { No group of AN } \\
\text { Not explicitly confirmed by } \\
\text { questionnaire or interview } \\
\text { Under nutritional markers: NR (but } \\
\text { blood glucose and lipid profile assessed) }\end{array}$ & $\begin{array}{l}\text { Amenorrhea: NR } \\
\text { Weight gain resistance/desire: NR } \\
\text { Healthy, associated pathology: NR } \\
\text { Physical activity: NR }\end{array}$ & $1,2,5$ \\
\hline $\begin{array}{l}\text { Mazzeo et } \\
\text { al., } 2004{ }^{(42)}\end{array}$ & $\begin{array}{l}\text { Males: } \\
\text { CT: } n=158 \text {; NR but probably 29- } \\
69 \mathrm{yr} ; 22.5 \pm 2.1 \mathrm{~kg} \cdot \mathrm{m}^{-2}\end{array}$ & $\begin{array}{l}\text { Presence of terminology (but "persistent } \\
\text { thinness" preferentially used) } \\
\text { Thinness threshold: } 1-4 \text { (1: very thin, } 9 \\
\text { very large) on silhouette ratings }\end{array}$ & $\begin{array}{l}\text { Considered (DSM-III-R) } \\
\text { No group of AN } \\
\text { Confirmed by interview (Structured }\end{array}$ & $\begin{array}{l}\text { Criterion of amenorrhea: NA (males) } \\
\text { Weight gain resistance/desire: NR } \\
\text { Healthy, associated pathology: NR } \\
\text { Physical activity: NR }\end{array}$ & 11 \\
\hline
\end{tabular}




\begin{tabular}{|c|c|c|c|c|c|}
\hline & $\begin{array}{l}\mathrm{C}: \mathrm{n}=915 ; \text { NR but probably } 29-69 \\
\mathrm{yr} ; 27.6 \pm 4.2 \mathrm{~kg} \cdot \mathrm{m}^{-2}\end{array}$ & $\begin{array}{l}\text { Consideration of personal weight history } \\
\text { (persistent thinness with consideration of } \\
\text { childhood, adolescence and adulthood) }\end{array}$ & $\begin{array}{l}\text { Clinical Interview for DSM-III-R) } \\
\text { Under nutritional markers: NR }\end{array}$ & & \\
\hline $\begin{array}{l}\text { Tagami et al., } \\
2004^{(37)}\end{array}$ & $\begin{array}{l}\text { Females: } \\
\text { CT: } n=6 ; 27.5 \pm 4.2 \mathrm{yr} ; 17.7 \pm 0.5 \\
\text { kg.m-2 } \\
\text { C: } n=16 ; 25.7 \pm 2.9 \mathrm{yr} ; 20.3 \pm 1.5 \\
\text { kg.m }{ }^{-2} \\
\text { AN: } n=31 ; 25.5 \pm 8.1 \mathrm{yr} ; 14.0 \pm \\
2.5 \mathrm{~kg} \cdot \mathrm{m}^{-2} \\
\text { (AN: probably restrictive-type) }\end{array}$ & $\begin{array}{l}\text { Presence of terminology } \\
\text { Thinness threshold: BMI }<18.0 \mathrm{~kg} \cdot \mathrm{m}^{-2} \\
\text { No apparent consideration of weight } \\
\text { history }\end{array}$ & $\begin{array}{l}\text { Considered } \\
\text { Implicitly confirmed by the presence of } \\
\text { a group of AN (DSM-IV) } \\
\text { Not explicitly confirmed by } \\
\text { questionnaire or interview } \\
\text { Under nutritional markers: NR }\end{array}$ & $\begin{array}{l}\text { No amenorrhea } \\
\text { Weight gain resistance/desire: NR } \\
\text { Healthy, absence of associated } \\
\text { pathology } \\
\text { Physical activity: NR }\end{array}$ & 2,3 \\
\hline $\begin{array}{l}\text { Miljic et al., } \\
2006^{(38)}\end{array}$ & $\begin{array}{l}\text { Females: } \\
\text { CT: } n=10 ; 22.5 \pm 4.4 \mathrm{yr} ; 17.6 \pm 1.3 \\
\text { kg.m }{ }^{-2} \\
\text { AN: } n=9 ; 25.1 \pm 5.1 \mathrm{yr} ; 12.0 \pm 1.2 \\
\text { kg.m }{ }^{-2} \\
\text { (AN: restrictive and binge } \\
\text { eating/purging type) }\end{array}$ & $\begin{array}{l}\text { Presence of terminology } \\
\text { Thinness threshold: NR (but subnormal } \\
\text { bodyweight } 51.4 \pm 7.6 \mathrm{~kg}(45-60 \mathrm{~kg} \text { ) and } \\
\text { BMI } 17.6 \pm 1.3 \mathrm{~kg} \cdot \mathrm{m}^{-2}\left(16.6-19.3 \mathrm{~kg} \cdot \mathrm{m}^{-2}\right) \text { ) } \\
\text { Consideration of personal weight history } \\
\text { (without history of weight loss) }\end{array}$ & $\begin{array}{l}\text { Considered } \\
\text { Implicitly confirmed by the presence of } \\
\text { a group of AN (DSM-IV) } \\
\text { Not explicitly confirmed by } \\
\text { questionnaire or interview } \\
\text { Under nutritional markers: NR }\end{array}$ & $\begin{array}{l}\text { No amenorrhea } \\
\text { Weight gain resistance/desire: NR } \\
\text { Healthy, associated pathology: NR } \\
\text { Physical activity: NR }\end{array}$ & 3,4 \\
\hline $\begin{array}{l}\text { Bossu et al., } \\
2007 \text { (7) }\end{array}$ & $\begin{array}{l}\text { Females: } \\
\text { CT: } n=7 ; \text { NR but } 18-26 \mathrm{yr} ; 16.1 \pm \\
0.6 \mathrm{~kg} \cdot \mathrm{m}^{-2} \\
\text { C: } \mathrm{n}=7 ; \mathrm{NR} \text { but } 18-26 \mathrm{yr} ; 21.2 \pm \\
0.8 \mathrm{~kg} \cdot \mathrm{m}^{-2} \\
\text { AN: } \mathrm{n}=6 \text {; NR but } 18-26 \mathrm{yr} ; 15.8 \pm \\
0.8 \mathrm{~kg} \cdot \mathrm{m}^{-2} \\
\text { (AN: restrictive-type) }\end{array}$ & $\begin{array}{l}\text { Presence of terminology } \\
\text { Thinness threshold: BMI: } 14.5-16.5 \mathrm{~kg} \cdot \mathrm{m}^{-2} \\
\text { Consideration of personal weight history } \\
\text { (stable throughout the post-pubertal period } \\
\text { and weight history retrospectively } \\
\text { reconstituted from birth to } 18 \text { years) }\end{array}$ & $\begin{array}{l}\text { Considered } \\
\text { Implicitly confirmed by the presence of } \\
\text { a group of AN (DSM-IV) } \\
\text { Not explicitly confirmed by } \\
\text { questionnaire or interview } \\
\text { Under nutritional markers: NR }\end{array}$ & $\begin{array}{l}\text { No amenorrhea } \\
\text { Weight gain resistance/desire: } \\
\text { mentioned (desire for weight gain as a } \\
\text { main reason for medical consultation) } \\
\text { Healthy, absence of associated } \\
\text { pathology } \\
\text { Absence of over-exercising }\end{array}$ & $\begin{array}{l}1,2 \\
3,11\end{array}$ \\
\hline $\begin{array}{l}\text { Germain et } \\
\text { al., } 2007^{(9)}\end{array}$ & $\begin{array}{l}\text { Females: } \\
\text { CT: } n=10 ; 20.2 \pm 3.8 \mathrm{yr} ; 15.7 \pm 0.6 \\
\text { kg.m-2 } \\
\text { C: } n=7 ; 23 \pm 2.1 \mathrm{yr} ; 20.4 \pm 0.8 \\
\text { kg.m }{ }^{-2} \\
\text { AN: } n=12 ; 20.7 \pm 4.2 \mathrm{yr} ; 15.2 \pm \\
1.4 \mathrm{~kg} \cdot \mathrm{m}^{-2} \\
\text { (AN: probably restrictive-type) }\end{array}$ & $\begin{array}{l}\text { Presence of terminology } \\
\text { Thinness threshold: BMI: } 14.5-16.5 \mathrm{~kg} \cdot \mathrm{m}^{-2} \\
\text { Consideration of personal weight history } \\
\text { (stable throughout the post-pubertal } \\
\text { period) }\end{array}$ & $\begin{array}{l}\text { Considered } \\
\text { Implicitly confirmed by the presence of } \\
\text { a group of AN (DSM-IV) } \\
\text { Not explicitly confirmed by } \\
\text { questionnaire or interview } \\
\text { Under nutritional markers: NR }\end{array}$ & $\begin{array}{l}\text { No amenorrhea } \\
\text { Weight gain resistance/desire: } \\
\text { mentioned (desire for weight gain as a } \\
\text { main reason for medical consultation) } \\
\text { Healthy, absence of associated } \\
\text { pathology } \\
\text { Physical activity: NR }\end{array}$ & $\begin{array}{l}1,2 \\
3,4\end{array}$ \\
\hline $\begin{array}{l}\text { Marra et al., } \\
2007^{(17)}\end{array}$ & $\begin{array}{l}\text { Females: } \\
\text { CT: } n=20 ; 22.5 \pm 5.8 \mathrm{yr} ; 17.2 \pm 1.0 \\
\mathrm{~kg} \cdot \mathrm{m}^{-2} \\
\mathrm{C}: \mathrm{n}=20 ; 22.0 \pm 3.7 \mathrm{yr} ; 21.7 \pm 2.4 \\
\mathrm{~kg} \cdot \mathrm{m}^{-2} \\
\text { AN: } \mathrm{n}=20 ; 18.8 \pm 3.4 \mathrm{yr} ; 15.1 \pm \\
1.6 \mathrm{~kg} \cdot \mathrm{m}^{-2} \\
\text { (AN: restrictive-type) }\end{array}$ & $\begin{array}{l}\text { Presence of terminology } \\
\text { Thinness threshold: NR } \\
\text { Consideration of personal weight history } \\
\text { (bodyweight that has always been in the } \\
\text { lower percentiles for age, gender and } \\
\text { ethnicity) }\end{array}$ & $\begin{array}{l}\text { Considered } \\
\text { Implicitly confirmed by the presence of } \\
\text { a group of AN (DSM-IV) } \\
\text { Not explicitly confirmed by } \\
\text { questionnaire or interview } \\
\text { Under nutritional markers: NR (but } \\
\text { normal thyroid functions seem to be } \\
\text { assessed) }\end{array}$ & $\begin{array}{l}\text { No amenorrhea } \\
\text { Weight gain resistance/desire: NR } \\
\text { Healthy, absence of associated } \\
\text { pathology } \\
\text { Absence of over-exercising }\end{array}$ & 1,2 \\
\hline
\end{tabular}




\begin{tabular}{|c|c|c|c|c|c|}
\hline $\begin{array}{l}\text { Galusca et } \\
\text { al., } 2008^{(28)}\end{array}$ & $\begin{array}{l}\text { Females: } \\
\text { CT: } n=25 ; 23.1 \pm 6.0 \mathrm{yr} ; 15.8 \pm 0.5 \\
\text { kg.m-2 } \\
\text { C: } n=28 ; 23.9 \pm 7.4 \mathrm{yr} ; 20.7 \pm 2.1 \\
\text { kg.m }{ }^{-2} \\
\text { AN: } n=44 ; 23.4 \pm 8.0 \mathrm{yr} ; \text { AN: } 15.5 \\
\pm 0.7 \text { kg.m }{ }^{-2} \\
\text { (AN: restrictive-type) }\end{array}$ & $\begin{array}{l}\text { Presence of terminology } \\
\text { Thinness threshold: BMI: } 12.0-16.5 \mathrm{~kg} \cdot \mathrm{m}^{-2} \\
\text { Consideration of personal weight history } \\
\text { (stable throughout the growth period until } \\
\text { the age of 18) }\end{array}$ & $\begin{array}{l}\text { Considered } \\
\text { Implicitly confirmed by the presence of } \\
\text { a group of AN (DSM-IV) } \\
\text { Not explicitly confirmed by } \\
\text { questionnaire or interview } \\
\text { Under nutritional markers: NR }\end{array}$ & $\begin{array}{l}\text { No amenorrhea } \\
\text { Weight gain resistance/desire: } \\
\text { mentioned (desire for weight gain as a } \\
\text { main reason for medical consultation) } \\
\text { Healthy, absence of associated } \\
\text { pathology } \\
\text { Absence of over-exercising }\end{array}$ & $2,3,5$ \\
\hline $\begin{array}{l}\text { Fernández- } \\
\text { García et al., } \\
2009^{(24)}\end{array}$ & $\begin{array}{l}\text { Females: } \\
\text { CT: } n=22 ; 19.7 \pm 5.3 \mathrm{yr} ; 16.7 \pm 1.0 \\
\text { kg.m-2 } \\
\text { C: } n=20 ; 19.3 \pm 1.6 \mathrm{yr} ; 22.3 \pm 1.6 \\
\text { kg.m }{ }^{-2} \\
\text { AN: } n=25 ; \text { NR for restrictive-type; } \\
16.1 \pm 1.5 \text { kg.m }{ }^{-2} \\
\text { (AN: restrictive-type) }\end{array}$ & $\begin{array}{l}\text { Absence of terminology } \\
\text { Thinness threshold: BMI }<18.5 \mathrm{~kg} . \mathrm{m}^{-2} \\
\text { No apparent consideration of weight } \\
\text { history (but after } 5 \text { years of follow-up, } \\
\text { none presented any criteria for eating } \\
\text { disorders) }\end{array}$ & $\begin{array}{l}\text { Considered } \\
\text { Implicitly confirmed by the presence of } \\
\text { a group of AN (DSM-IV) } \\
\text { Not explicitly confirmed by } \\
\text { questionnaire or interview } \\
\text { Under nutritional markers: NR }\end{array}$ & $\begin{array}{l}\text { No amenorrhea } \\
\text { Weight gain resistance/desire: NR } \\
\text { Healthy, associated pathology: NR } \\
\text { Physical activity: NR }\end{array}$ & $2,3,5$ \\
\hline $\begin{array}{l}\text { Germain et } \\
\text { al., } 2009^{(21)}\end{array}$ & $\begin{array}{l}\text { Females: } \\
\text { CT: } n=9 ; 24.1 \pm 3.6 \mathrm{yr} ; 16.1 \pm 0.3 \\
\mathrm{~kg} \cdot \mathrm{m}^{-2} \\
\text { C: } \mathrm{n}=10 ; 23.1 \pm 4.4 \mathrm{yr} ; 20.5 \pm 1.3 \\
\mathrm{~kg} \cdot \mathrm{m}^{-2} \\
\text { AN: } \mathrm{n}=15 ; 20.4 \pm 5.0 \mathrm{yr} ; 14.8 \pm \\
0.4 \mathrm{~kg} \cdot \mathrm{m}^{-2} \\
\text { (AN: restrictive-type) }\end{array}$ & $\begin{array}{l}\text { Presence of terminology } \\
\text { Thinness threshold: BMI }<16.5 \mathrm{~kg} \cdot \mathrm{m}^{-2} \\
\text { Consideration of personal weight history } \\
\text { (stable throughout the growth period) }\end{array}$ & $\begin{array}{l}\text { Considered } \\
\text { Implicitly confirmed by the presence of } \\
\text { a group of AN (DSM-IV) } \\
\text { Not explicitly confirmed by } \\
\text { questionnaire or interview } \\
\text { Under nutritional markers: NR }\end{array}$ & $\begin{array}{l}\text { No amenorrhea } \\
\text { Weight gain resistance/desire: } \\
\text { mentioned (a stated desire for weight } \\
\text { gain) } \\
\text { Healthy, absence of associated } \\
\text { pathology } \\
\text { Physical activity: NR }\end{array}$ & $2,3,4$ \\
\hline $\begin{array}{l}\text { Marra et al., } \\
2009^{(25)}\end{array}$ & $\begin{array}{l}\text { Females: } \\
\text { CT: } n=10 ; 19.4 \pm 2.4 \mathrm{yr} ; 16.8 \pm 1 \\
\text { kg.m }{ }^{-2} \\
\text { C: } n=30 ; 20.0 \pm 2.1 \mathrm{yr} ; 22.5 \pm 2.8 \\
\text { kg.m }{ }^{-2} \\
\text { AN: } n=30 ; 19.0 \pm 2.0 \mathrm{yr} ; 16.7 \pm \\
0.5 \mathrm{~kg} \cdot \mathrm{m}^{-2} \\
\text { (AN type: NR) }\end{array}$ & $\begin{array}{l}\text { Presence of terminology } \\
\text { Thinness threshold: BMI }<18.5 \mathrm{~kg} \cdot \mathrm{m}^{-2} \\
\text { No apparent consideration of weight } \\
\text { history }\end{array}$ & $\begin{array}{l}\text { Considered } \\
\text { Implicitly confirmed by the presence of } \\
\text { a group of AN } \\
\text { Not explicitly confirmed by } \\
\text { questionnaire or interview } \\
\text { Under nutritional markers: NR }\end{array}$ & $\begin{array}{l}\text { No amenorrhea } \\
\text { Weight gain resistance/desire: NR } \\
\text { Healthy, absence of associated } \\
\text { pathology } \\
\text { Absence of over-exercising }\end{array}$ & 2 \\
\hline $\begin{array}{l}\text { Hasegawa et } \\
\text { al., } 2011 \text { (26) }\end{array}$ & $\begin{array}{l}\text { Females: } \\
\text { CT: } n=20 ; 23.2 \pm 2.3 \text { yr; } 17.6 \pm 0.8 \\
\text { kg.m }{ }^{-2} \\
\text { C: } n=20 ; 23.1 \pm 2.1 \mathrm{yr} ; 21.9 \pm 1.2 \\
\text { kg.m }{ }^{-2}\end{array}$ & $\begin{array}{l}\text { Presence of terminology (but "lean" term } \\
\text { preferentially used) } \\
\text { Thinness threshold: BMI }<18.5 \mathrm{~kg} \cdot \mathrm{m}^{-2} \\
\text { No apparent consideration of weight } \\
\text { history }\end{array}$ & $\begin{array}{l}\text { Considered } \\
\text { No group of AN } \\
\text { Not explicitly confirmed by } \\
\text { questionnaire or interview } \\
\text { Under nutritional markers: NR }\end{array}$ & $\begin{array}{l}\text { No amenorrhea } \\
\text { Weight gain resistance/desire: NR } \\
\text { Healthy, absence of associated } \\
\text { pathology } \\
\text { Physical activity: NR }\end{array}$ & $1,2,3$ \\
\hline $\begin{array}{l}\text { Galusca et } \\
\text { al., } 2012^{(22)}\end{array}$ & $\begin{array}{l}\text { Females: } \\
\text { CT: } n=14 ; 23.7 \pm 6^{\S} \mathrm{yr} ; 16.0 \pm 0.4^{\S} \\
\text { kg.m }{ }^{-2} \\
\text { C: } n=10 ; 23.1 \pm 5^{\S} \mathrm{yr} ; 20.8 \pm 0.6^{\S} \\
\mathrm{kg} \cdot \mathrm{m}^{-2}\end{array}$ & $\begin{array}{l}\text { Presence of terminology } \\
\text { Thinness threshold: BMI }<16.5 \mathrm{~kg} \cdot \mathrm{m}^{-2} \\
\text { Consideration of personal weight history } \\
\text { (stable throughout the growth period) }\end{array}$ & $\begin{array}{l}\text { Considered } \\
\text { Implicitly confirmed by the presence of } \\
\text { a group of AN (DSM-IV) } \\
\text { Not explicitly confirmed by }\end{array}$ & $\begin{array}{l}\text { No amenorrhea } \\
\text { Weight gain resistance/desire: } \\
\text { mentioned (a stated desire for weight } \\
\text { gain) } \\
\text { Healthy, absence of associated }\end{array}$ & $2,3,4$ \\
\hline
\end{tabular}




\begin{tabular}{|c|c|c|c|c|c|}
\hline & $\begin{array}{l}\text { AN: } \mathrm{n}=19 ; 23.2 \pm 8^{\S} \mathrm{yr} ; 15.3 \pm \\
0.4^{\S} \mathrm{kg} \cdot \mathrm{m}^{-2} \\
\text { (AN: restrictive-type) }\end{array}$ & & $\begin{array}{l}\text { questionnaire or interview } \\
\text { Under nutritional markers: NR }\end{array}$ & $\begin{array}{l}\text { pathology } \\
\text { Absence of over-exercising }\end{array}$ & \\
\hline $\begin{array}{l}\text { Santonicola } \\
\text { et al., } 2012 \\
\text { (46) }\end{array}$ & $\begin{array}{l}\text { Females and males (not clearly } \\
\text { reported): } \\
\text { CT: } n=9 ; 24.9 \pm 6.6 \mathrm{yr} ; \mathrm{NR} \\
\mathrm{C}: \mathrm{n}=22 ; 23.7 \pm 3.3 \mathrm{yr} ; \mathrm{NR} \\
\text { AN: } \mathrm{n}=20 ; 22.5 \pm 4.2 \mathrm{yr} ; \mathrm{NR} \\
\text { (AN: probably restrictive-type) }\end{array}$ & $\begin{array}{l}\text { Presence of terminology } \\
\text { Thinness threshold: NR (but severely } \\
\text { underweight) } \\
\text { Consideration of personal weight history } \\
\text { (stable throughout the post-pubertal } \\
\text { period) }\end{array}$ & $\begin{array}{l}\text { Considered (DSM-IV) } \\
\text { Implicitly confirmed by the presence of } \\
\text { a group of AN (DSM-IV) } \\
\text { Confirmed by interview (to detect } \\
\text { potential lifetime eating disorders in } \\
\text { accordance with the criteria of the DSM- } \\
\text { IV) } \\
\text { Under nutritional markers: NR }\end{array}$ & $\begin{array}{l}\text { No amenorrhea } \\
\text { Weight gain resistance/desire: } \\
\text { mentioned (desire for weight gain as a } \\
\text { main reason for medical consultation) } \\
\text { Healthy, absence of associated } \\
\text { pathology } \\
\text { Physical activity: NR }\end{array}$ & 13 \\
\hline $\begin{array}{l}\text { Pasanisi et } \\
\text { al., } 2013^{(20)}\end{array}$ & $\begin{array}{l}\text { Females: } \\
\text { CT: } n=7 ; 21.7 \pm 3.6 \mathrm{yr} ; 16.2 \pm 0.9 \\
\text { kg.m }{ }^{-2} \\
\text { C: } n=20 ; 25.6 \pm 3.9 \mathrm{yr} ; 21.7 \pm 2.4 \\
\text { kg.m }{ }^{-2} \\
\text { AN: } n=7 ; 23.4 \pm 4.5 \mathrm{yr} ; 15.3 \pm 0.8 \\
\text { kg.m }{ }^{-2} \\
\text { (AN: restrictive-type) }\end{array}$ & $\begin{array}{l}\text { Presence of terminology } \\
\text { Thinness threshold: NR } \\
\text { No apparent consideration of weight } \\
\text { history }\end{array}$ & $\begin{array}{l}\text { Considered } \\
\text { Implicitly confirmed by the presence of } \\
\text { a group of AN (DSM-IV) } \\
\text { Not explicitly confirmed by } \\
\text { questionnaire or interview } \\
\text { Under nutritional markers: NR (but } \\
\text { normal thyroid function) }\end{array}$ & $\begin{array}{l}\text { No amenorrhea } \\
\text { Weight gain resistance/desire: NR } \\
\text { Healthy, absence of associated } \\
\text { pathology } \\
\text { Physical activity: NR }\end{array}$ & $\begin{array}{l}1,2 \\
10\end{array}$ \\
\hline $\begin{array}{l}\text { Paschalis et } \\
\text { al., } 2013^{(39)}\end{array}$ & $\begin{array}{l}\text { Females: } \\
\text { CT: } n=8 ; 21.4 \pm 1.1 \mathrm{yr} ; 17.3 \pm 0.6 \\
\text { kg.m-2 } \\
\text { C: } n=12 ; 20.2 \pm 1.4 \mathrm{yr} ; 22.0 \pm 1.0 \\
\text { kg.m }{ }^{-2}\end{array}$ & $\begin{array}{l}\text { Absence of terminology } \\
\text { Thinness threshold: NR (but groups } \\
\text { constituted according to BMI) } \\
\text { Consideration of personal weight history } \\
\text { (stable at their anthropometric } \\
\text { characteristics for at least the last } 2 \text { years) }\end{array}$ & $\begin{array}{l}\text { NR } \\
\text { No group of AN } \\
\text { Not explicitly confirmed by } \\
\text { questionnaire or interview } \\
\text { Under nutritional markers: NR }\end{array}$ & $\begin{array}{l}\text { No amenorrhea } \\
\text { Weight gain resistance/desire: NR } \\
\text { Healthy, absence of associated } \\
\text { pathology } \\
\text { Absence of over-exercising ( } \leq 1 \text { hour per } \\
\text { week on sport activities) }\end{array}$ & 6 \\
\hline $\begin{array}{l}\text { Germain et } \\
\text { al., } 2014^{(10)}\end{array}$ & $\begin{array}{l}\text { Females: } \\
\text { CT: } n=8 ; 21.6 \pm 5.4 \text { yr; } 17.1 \pm 0.8 \\
\text { kg.m-2 } \\
\text { C: } n=8 ; 22.1 \pm 2.3 \text { yr; } 22.1 \pm 0.8 \\
\text { kg.m }{ }^{-2}\end{array}$ & $\begin{array}{l}\text { Presence of terminology } \\
\text { Thinness threshold: BMI: } 13-17.5 \mathrm{~kg} \cdot \mathrm{m}^{-2} \\
\text { Consideration of personal weight history } \\
\text { (stable throughout the post-pubertal } \\
\text { period) }\end{array}$ & $\begin{array}{l}\text { Considered } \\
\text { No group of AN } \\
\text { Confirmed by questionnaires (DEBQ } \\
\text { and EDE - no reported thresholds) } \\
\text { Normal nutritional markers (normal } \\
\text { IGF-1, estradiol, } \mathrm{FT}_{3} \text {, mean cortisol and } \\
\text { non-blunted leptin) }\end{array}$ & $\begin{array}{l}\text { No amenorrhea } \\
\text { Weight gain resistance/desire: } \\
\text { mentioned (recruited among outpatients } \\
\text { consulting for bodyweight gain desire) } \\
\text { Healthy, absence of associated } \\
\text { pathology } \\
\text { Absence of over-exercising (according } \\
\text { to the MOSPA questionnaire) }\end{array}$ & $\begin{array}{l}1,2 \\
3,4 \\
7,11\end{array}$ \\
\hline $\begin{array}{l}\text { Galusca et } \\
\text { al., } 2015^{(23)}\end{array}$ & $\begin{array}{l}\text { Females: } \\
\text { CT: } n=22 ; 23.2 \pm 2.3 \mathrm{yr} ; 15.9 \pm 0.5 \\
\text { kg.m }{ }^{-2} \\
\text { C: } n=14 ; 22.6 \pm 6.0 \mathrm{yr} ; 21.6 \pm 1.1 \\
\text { kg.m }{ }^{-2} \\
\text { AN: } n=23 ; 22.5 \pm 6.2 \mathrm{yr} ; 14.6 \pm \\
2.4 \mathrm{~kg} \cdot \mathrm{m}^{-2} \\
\text { (AN: restrictive-type) }\end{array}$ & $\begin{array}{l}\text { Presence of terminology } \\
\text { Thinness threshold: BMI < } 16.5 \mathrm{~kg} . \mathrm{m}^{-2} \\
\text { Consideration of personal weight history } \\
\text { (stable throughout the growth period) }\end{array}$ & $\begin{array}{l}\text { Considered } \\
\text { Implicitly confirmed by the presence of } \\
\text { a group of AN (DSM-IV) } \\
\text { Not explicitly confirmed by } \\
\text { questionnaire or interview } \\
\text { Under nutritional markers: NR }\end{array}$ & $\begin{array}{l}\text { No amenorrhea } \\
\text { Weight gain resistance/desire: } \\
\text { mentioned (a stated desire for weight } \\
\text { gain) } \\
\text { Healthy, absence of associated } \\
\text { pathology } \\
\text { Absence of over-exercising (according } \\
\text { to the MOSPA questionnaire) }\end{array}$ & $2,3,4$ \\
\hline
\end{tabular}




\begin{tabular}{|c|c|c|c|c|c|}
\hline $\begin{array}{l}\text { Germain et } \\
\text { al., } 2016^{(27)}\end{array}$ & $\begin{array}{l}\text { Females: } \\
\text { CT: } n=10 ; 20.6 \pm 6.6 \mathrm{yr} ; 15.9 \pm 0.9 \\
\mathrm{~kg} \cdot \mathrm{m}^{-2} \\
\mathrm{C}: \mathrm{n}=10 ; 22.7 \pm 1.6 \mathrm{yr} ; 21.4 \pm 1.6 \\
\mathrm{~kg} \cdot \mathrm{m}^{-2} \\
\text { AN: } \mathrm{n}=10 ; 21.6 \pm 4.7 \mathrm{yr} ; 15.1 \pm \\
2.5 \mathrm{~kg} \cdot \mathrm{m}^{-2} \\
\text { (AN: restrictive-type) }\end{array}$ & $\begin{array}{l}\text { Presence of terminology } \\
\text { Thinness threshold: BMI }<17 \mathrm{~kg} . \mathrm{m}^{-2} \\
\text { Consideration of personal weight history } \\
\text { (stable throughout the growth period) }\end{array}$ & $\begin{array}{l}\text { Considered } \\
\text { Implicitly confirmed by the presence of } \\
\text { a group of AN (DSM-IV) } \\
\text { Not explicitly confirmed by } \\
\text { questionnaire or interview } \\
\text { Under nutritional markers: NR }\end{array}$ & $\begin{array}{l}\text { No amenorrhea } \\
\text { Weight gain resistance/desire: } \\
\text { mentioned (a stated desire for weight } \\
\text { gain) } \\
\text { Healthy, absence of associated } \\
\text { pathology } \\
\text { Physical activity: NR }\end{array}$ & 2,3 \\
\hline $\begin{array}{l}\text { Gunes et al., } \\
2016^{(47)}\end{array}$ & $\begin{array}{l}\text { CT (16 females, } 8 \text { males }): \mathrm{n}=24 \\
22.1 \pm 3.7 \mathrm{yr} ; 17.4 \pm 1.2 \mathrm{~kg} \cdot \mathrm{m}^{-2} \\
\text { C }(9 \text { females, } 15 \text { males }): \mathrm{n}=24 \\
23.5 \pm 4.0 \mathrm{yr} ; 22.1 \pm 2.4 \mathrm{~kg} \cdot \mathrm{m}^{-2}\end{array}$ & $\begin{array}{l}\text { Presence of terminology } \\
\text { Thinness threshold: BMI }<18.5 \mathrm{~kg} . \mathrm{m}^{-2} \\
\text { Consideration of personal weight history } \\
\text { (stable during the post-pubertal period) }\end{array}$ & $\begin{array}{l}\text { NR } \\
\text { No group of AN } \\
\text { Not explicitly confirmed by } \\
\text { questionnaire or interview } \\
\text { Under nutritional markers: NR }\end{array}$ & $\begin{array}{l}\text { No amenorrhea } \\
\text { Weight gain resistance/desire: } \\
\text { mentioned (desire for weight gain as a } \\
\text { main reason for medical consultation) } \\
\text { Healthy, absence of associated } \\
\text { pathology } \\
\text { Absence of over-exercising }\end{array}$ & 8 \\
\hline $\begin{array}{l}\text { Ling et al., } \\
2016^{(31)}\end{array}$ & $\begin{array}{l}\text { Females: } \\
\text { CT: } \mathrm{n}=15 \text {; NR (design) but 18-35 } \\
\text { yr; NR (design) } 13-17.5 \mathrm{~kg} \cdot \mathrm{m}^{-2} \\
\text { C: } \mathrm{n}=15 ; \mathrm{NR} \text { (design) but } 18-35 \\
\text { yr; NR (design) but } 20-25 \mathrm{~kg} \cdot \mathrm{m}^{-2} \\
\text { Males: } \\
\text { CT: } \mathrm{n}=15 \text {; NR (design) but } 18-35 \\
\text { yr; NR (design) } 13-18.5 \mathrm{~kg} \cdot \mathrm{m}^{-2} \\
\text { C: } \mathrm{n}=15 ; \mathrm{NR} \text { (design) but } 18-35 \\
\text { yr; NR (design) but } 20-25 \mathrm{~kg} \cdot \mathrm{m}^{-2}\end{array}$ & $\begin{array}{l}\text { Presence of terminology } \\
\text { Thinness threshold: BMI: } 13-17.5 \mathrm{~kg} \cdot \mathrm{m}^{-2} \\
\text { (females), } 13-18.5 \mathrm{~kg} \cdot \mathrm{m}^{-2} \text { (males) } \\
\text { Consideration of personal weight history } \\
\text { (stable for post-pubertal and at least } 3 \\
\text { months) }\end{array}$ & $\begin{array}{l}\text { Considered (DSM-IV) } \\
\text { No group of AN } \\
\text { Confirmed by questionnaires (DEBQ, } \\
\text { EDE, EDI, and BSQ - no reported } \\
\text { thresholds) } \\
\text { Normal nutritional markers (normal } \\
\text { IGF-1, estradiol, FT } \text { F, }_{3} \text {, mean cortisol and } \\
\text { non-blunted leptin) }\end{array}$ & $\begin{array}{l}\text { No amenorrhea } \\
\text { Weight gain resistance/desire: } \\
\text { mentioned (recruited among outpatients } \\
\text { consulting for bodyweight gain desire) } \\
\text { Healthy, absence of associated } \\
\text { pathology } \\
\text { Absence of over-exercising (according } \\
\text { to the MOSPA questionnaire and } \leq 3 \\
\text { sessions per week) }\end{array}$ & $\begin{array}{l}1,2 \\
3,4 \\
5,6 \\
7,11\end{array}$ \\
\hline $\begin{array}{l}\text { Estour et al., } \\
2017^{(8)}\end{array}$ & $\begin{array}{l}\text { Females: } \\
\text { CT: } n=56 ; 26.9 \pm 7.6 \mathrm{yr} ; 16.5 \pm 0.9 \\
\text { kg.m }{ }^{-2} \\
\text { C: } n=54 ; 23.4 \pm 4.1 \mathrm{yr} ; 20.9 \pm 2.2 \\
\text { kg.m }{ }^{-2} \\
\text { AN: } n=40 ; 25.0 \pm 6.5 \mathrm{yr} ; 16.0 \pm \\
0.8 \mathrm{~kg} \cdot \mathrm{m}^{-2} \\
\text { (AN: restrictive-type) }\end{array}$ & $\begin{array}{l}\text { Presence of terminology } \\
\text { Thinness threshold: BMI }<17.5 \mathrm{~kg} . \mathrm{m}^{-2} \\
\text { Consideration of personal weight history } \\
\text { (when available }(26 / 56 \mathrm{CT}) \text {, weight } \\
\text { history from birth to at least } 18 \text { years old } \\
\text { was retrospectively reconstituted) }\end{array}$ & $\begin{array}{l}\text { Considered } \\
\text { Implicitly confirmed by the presence of } \\
\text { a group of AN (DSM-IV) } \\
\text { Not explicitly confirmed by } \\
\text { questionnaire or interview } \\
\text { Under nutritional markers: NR }\end{array}$ & $\begin{array}{l}\text { No amenorrhea } \\
\text { Weight gain resistance/desire: } \\
\text { mentioned (desire for weight gain as a } \\
\text { main reason for medical consultation) } \\
\text { Healthy, absence of associated } \\
\text { pathology } \\
\text { Physical activity: NR }\end{array}$ & $\begin{array}{l}1,2 \\
3,5 \\
11\end{array}$ \\
\hline $\begin{array}{l}\text { Galusca et } \\
\text { al., } 2018^{(19)}\end{array}$ & $\begin{array}{l}\text { Females: } \\
\text { CT: }=10 ; 22.1 \pm 5.1 \mathrm{yr} ; 17.0 \pm 0.9 \\
\mathrm{~kg} \cdot \mathrm{m}^{-2} \\
\mathrm{C}: \mathrm{n}=10 ; 22.2 \pm 2.5 \mathrm{yr} ; 21.7 \pm 1.3 \\
\mathrm{~kg} \cdot \mathrm{m}^{-2}\end{array}$ & $\begin{array}{l}\text { Presence of terminology } \\
\text { Thinness threshold: BMI }<17.5 \mathrm{~kg} . \mathrm{m}^{-2} \\
\text { Consideration of personal weight history } \\
\text { (stable throughout the post-pubertal } \\
\text { period) }\end{array}$ & $\begin{array}{l}\text { Considered } \\
\text { No group of AN } \\
\text { Not explicitly confirmed by } \\
\text { questionnaire or interview } \\
\text { Normal nutritional markers (normal } \\
\text { IGF-1, 17 } \beta \text { estradiol, } \mathrm{FT}_{3} \text { ) }\end{array}$ & $\begin{array}{l}\text { Amenorrhea: NR } \\
\text { Weight gain resistance/desire: NR } \\
\text { Healthy, absence of associated } \\
\text { pathology } \\
\text { Physical activity: NR }\end{array}$ & $\begin{array}{l}1,2 \\
3,5 \\
6,7\end{array}$ \\
\hline $\begin{array}{l}\text { Florent et al., } \\
2019^{(41)}\end{array}$ & $\begin{array}{l}\text { Females: } \\
\text { CT: } \mathrm{n}=10 ; 22.4 \pm 2.5 \mathrm{yr} ; 17.1 \pm 0.9 \\
\mathrm{~kg} \cdot \mathrm{m}^{-2}\end{array}$ & $\begin{array}{l}\text { Presence of terminology } \\
\text { Thinness threshold: } \mathrm{BMI}<18.5 \mathrm{~kg} \cdot \mathrm{m}^{-2}\end{array}$ & $\begin{array}{l}\text { Considered (DSM-IV) } \\
\text { Implicitly confirmed by the presence of } \\
\text { a group of AN (DSM-IV) }\end{array}$ & $\begin{array}{l}\text { No amenorrhea } \\
\text { Weight gain resistance/desire: NR } \\
\text { Healthy, absence of associated }\end{array}$ & 11,14 \\
\hline
\end{tabular}




\begin{tabular}{|c|c|c|c|c|c|}
\hline & $\begin{array}{l}\text { C: } n=10 ; 21.8 \pm 2.2 \mathrm{yr} ; 21.9 \pm 1.3 \\
\text { kg.m-2 } \\
\text { AN: } n=10 ; 26.4 \pm 6.0 \mathrm{yr} ; 15.3 \pm \\
1.9 \mathrm{~kg} . \mathrm{m}^{-2} \\
\text { (AN: restrictive-type) }\end{array}$ & $\begin{array}{l}\text { No apparent consideration of weight } \\
\text { history }\end{array}$ & $\begin{array}{l}\text { Confirmed by questionnaire (TFEQ with } \\
\text { a cognitive restraint score } \geq 13 \text { ) } \\
\text { Under nutritional markers: NR }\end{array}$ & $\begin{array}{l}\text { pathology } \\
\text { Physical activity: NR }\end{array}$ & \\
\hline $\begin{array}{l}\text { Margaritelis } \\
\text { et al., } 2019 \\
\text { (40) }\end{array}$ & $\begin{array}{l}\text { Females: } \\
\text { CT: } n=12 ; 21.2 \pm 1.4 \text { yr; } 17.8 \pm 0.8 \\
\text { kg.m-2 } \\
\text { C: } n=14 ; 20.4 \pm 1.8 \text { yr; } 22.4 \pm 1.1 \\
\text { kg.m } m^{-2}\end{array}$ & $\begin{array}{l}\text { Absence of terminology } \\
\text { Thinness threshold: BMI }<20 \mathrm{~kg} \cdot \mathrm{m}^{-2} \text { and } \\
\text { body fat: } 10-20 \% \\
\text { Consideration of personal weight history } \\
\text { (bodyweight did not change more than } \pm 3 \\
\mathrm{~kg} \text { the last } 2 \text { years prior to participation in } \\
\text { the study) }\end{array}$ & $\begin{array}{l}\text { NR } \\
\text { No group of AN } \\
\text { Not explicitly confirmed by } \\
\text { questionnaire or interview } \\
\text { Under nutritional markers: NR }\end{array}$ & $\begin{array}{l}\text { No amenorrhea } \\
\text { Weight gain resistance/desire: NR } \\
\text { Healthy, absence of associated } \\
\text { pathology } \\
\text { Absence of over-exercising ( } \leq 1 \text { hour per } \\
\text { week on sport activities) }\end{array}$ & $1,3,6$ \\
\hline $\begin{array}{l}\text { Marra et al., } \\
2019^{(18)}\end{array}$ & $\begin{array}{l}\text { Males: } \\
\text { CT: } n=15 ; 23.3 \pm 5.2 \mathrm{yr} ; 17.9 \pm 0.6 \\
\text { kg.m }{ }^{-2} \\
\text { C: } n=18 ; 22.3 \pm 3.7 \mathrm{yr} ; 22.3 \pm 1.7 \\
\text { kg.m }{ }^{-2} \\
\text { AN: } n=17 ; 22.3 \pm 5.3 \mathrm{yr} ; \text { AN: } 17.1 \\
\pm 1.2 \text { kg.m } \\
\text { (AN: probably restrictive-type) }\end{array}$ & $\begin{array}{l}\text { Presence of terminology } \\
\text { Thinness threshold: NR } \\
\text { Consideration of personal weight history } \\
\text { (stable on time) }\end{array}$ & $\begin{array}{l}\text { Considered } \\
\text { Implicitly confirmed by the presence of } \\
\text { a group of AN (DSM-V) } \\
\text { Not explicitly confirmed by } \\
\text { questionnaire or interview } \\
\text { Under nutritional markers: NR }\end{array}$ & $\begin{array}{l}\text { Criterion of amenorrhea: NA (males) } \\
\text { Weight gain resistance/desire: NR } \\
\text { Healthy, absence of associated } \\
\text { pathology } \\
\text { Physical activity: NR }\end{array}$ & 1,2 \\
\hline $\begin{array}{l}\text { Riveros- } \\
\text { McKay et al., } \\
2019^{(30)}\end{array}$ & $\begin{array}{l}\text { Females: } \\
\text { CT: } \mathrm{n}=1325 ; 36.6 \pm 14.3 \mathrm{yr} ; 17.6 \\
\pm 0.9 \mathrm{~kg} \cdot \mathrm{m}^{-2} \\
\mathrm{C}: \mathrm{n}=5837 ; 52.0 \pm 16.7 \mathrm{yr} ; 27.0 \pm \\
7.9 \mathrm{~kg} . \mathrm{m}^{-2} \\
\text { AN type: NR } \\
\text { Males: } \\
\text { CT: } \mathrm{n}=297 ; 35.2 \pm 14.5 \mathrm{yr} ; 17.6 \pm \\
1.1 \mathrm{~kg} . \mathrm{m}^{-2} \\
\text { C: } \mathrm{n}=4596 ; 52.7 \pm 17.3 \mathrm{yr} ; 26.9 \pm \\
7.8 \mathrm{~kg} . \mathrm{m}^{-2} \\
\text { AN type: NR }\end{array}$ & $\begin{array}{l}\text { Presence of terminology (but } \\
\text { "persistent/healthy thinness" preferentially } \\
\text { used) } \\
\text { Thinness threshold: BMI }<18 \mathrm{~kg} . \mathrm{m}^{-2} \text { (but } \\
\text { a small number of individuals with a BMI } \\
\text { of } 19.0 \mathrm{~kg} \cdot \mathrm{m}^{-2} \text { were included as they had a } \\
\text { strong family history of thinness) } \\
\text { Consideration of personal weight history } \\
\text { (persistently thin/always thin throughout } \\
\text { life) }\end{array}$ & $\begin{array}{l}\text { Considered } \\
\text { Implicitly confirmed by the presence of } \\
\text { a group of AN } \\
\text { Confirmed by questionnaire (SCOFF } \\
\text { questionnaire - no reported thresholds) } \\
\text { Under nutritional markers: NR }\end{array}$ & $\begin{array}{l}\text { Amenorrhea: NR } \\
\text { Weight gain resistance/desire: NR } \\
\text { Healthy, absence of associated } \\
\text { pathology } \\
\text { Absence of over-exercising (excluded if } \\
\text { they exercised more than } 3 \text { times a week } \\
\text { or with an intensity exceeding } 6 \text { METs } \\
\text { for any duration or frequency) }\end{array}$ & 7 \\
\hline
\end{tabular}

Abbreviations: BMI: body mass index, SD: standard deviation, CT: constitutional thinness, NR: not reported, C: control subjects, AN: anorexia nervosa, BSQ: body shape questionnaire (52), DSM: diagnostic and statistical manual of mental disorder, NA: not applicable, TFEQ: three-factor eating questionnaire ${ }^{(48)}$, CIDI: composite international diagnostic interview ${ }^{(54)}$, DEBQ: dutch eating behavior questionnaire ${ }^{(49)}$, EDE: eating disorder examination questionnaire ${ }^{(50)}$, IGF-1: insulin-like growth factor-1, FT $_{3}$ : free triiodothyronine, MOSPA: monica optional study of physical activity ${ }^{(56)}$, EDI: eating disorder inventory questionnaire ${ }^{(51)}$, SCOFF questions: Do you make yourself Sick because you feel uncomfortably full? Do you worry you have lost Control over how much you eat? Have you recently lost more than One stone in a 3-month period? Do you believe yourself to be Fat when others say you are too thin? Would you say that Food dominates your life? ${ }^{(53)}$, METs: metabolic equivalents 
Areas of study: 1: Energy balance, 2: Body composition, 3: Hormonal, biochemical assays, 4: Appetite-regulating hormones, 5: Bone tissue / Bone markers, 6: Muscle tissue / Muscle function, 7: Genetics or omics approaches, 8: Ophthalmology, 9: Pregnancy, 10: Thermogenesis / Brown adipose tissue, 11: Psychological profile, 12: Cardiology, 13: Functional dyspepsia, 14: Neurology

"Terminology presence" means the mention of "constitutional(ly) thin(ness)/lean(ness)" crucial keywords

$\S:$ Type of values dispersion (SD or SEM) not clearly reported 
Table 2. Risks of bias

\begin{tabular}{|c|c|c|c|c|c|c|}
\hline Reference & $\begin{array}{l}\text { Random sequence } \\
\text { generation } \\
\text { (selection bias) }\end{array}$ & $\begin{array}{l}\text { Allocation } \\
\text { concealment } \\
\text { (selection bias) }\end{array}$ & $\begin{array}{l}\text { Blinding of } \\
\text { participants and } \\
\text { personnel } \\
\text { (performance bias) }\end{array}$ & $\begin{array}{l}\text { Blinding of outcome } \\
\text { assessment } \\
\text { (detection bias) }\end{array}$ & $\begin{array}{l}\text { Incomplete outcome } \\
\text { data } \\
\text { (attrition bias) }\end{array}$ & $\begin{array}{l}\text { Selective reporting } \\
\text { (reporting bias) }\end{array}$ \\
\hline Schneider et al., $1979^{(33)}$ & Moderate risk & NR & Low risk & Moderate risk & Moderate risk & Moderate risk \\
\hline Diaz et al., 1992 & Low risk & NR & High risk & High risk & High risk & Low risk \\
\hline Scalfi et al., $1992^{(16)}$ & Low risk & NR & High risk & Moderate risk & NR & Low risk \\
\hline Hinney et al., $1997^{(44)}$ & Low risk & NR & High risk & Low risk & Low risk & Low risk \\
\hline Petretta et al., $1997^{(35)}$ & Low risk & NR & High risk & Low risk & NR & Low risk \\
\hline Tolle et al., $2003^{(11)}$ & Moderate risk & NR & High risk & Low risk & Moderate risk & Low risk \\
\hline Bosy-Westphal et al., 2004 (45) & Low risk & NR & High risk & Low risk & High risk & Low risk \\
\hline Mazzeo et al., 2004 & Moderate risk & NR & High risk & High risk & Low risk & Low risk \\
\hline Tagami et al., $2004{ }^{(37)}$ & Low risk & NR & High risk & Low risk & Moderate risk & Low risk \\
\hline Miljic et al., $2006^{(38)}$ & Moderate risk & NR & High risk & Low risk & Moderate risk & Low risk \\
\hline Bossu et al., $2007^{(7)}$ & Low risk & NR & High risk & Moderate risk & NR & Low risk \\
\hline Germain et al., $2007^{(9)}$ & Low risk & NR & High risk & Low risk & NR & Low risk \\
\hline Marra et al., $2007^{(17)}$ & Moderate risk & NR & High risk & Low risk & NR & Low risk \\
\hline Galusca et al., $2008^{(28)}$ & Low risk & NR & High risk & Low risk & NR & Low risk \\
\hline Fernández-García et al., $2009^{(24)}$ & Low risk & NR & High risk & Low risk & Moderate risk & Low risk \\
\hline Marra et al., $2009^{(25)}$ & Low risk & NR & High risk & Low risk & NR & Low risk \\
\hline Hasegawa et al., $2011^{(26)}$ & Low risk & NR & High risk & Low risk & NR & Low risk \\
\hline Galusca et al., $2012^{(22)}$ & Low risk & NR & High risk & Low risk & NR & Low risk \\
\hline Santonicola et al., $2012^{(46)}$ & Moderate risk & NR & High risk & High risk & NR & Low risk \\
\hline Pasanisi et al., $2013^{(20)}$ & Moderate risk & NR & High risk & Low risk & Moderate risk & Low risk \\
\hline Paschalis et al., $2013^{(39)}$ & Moderate risk & NR & High risk & Moderate risk & NR & Low risk \\
\hline Germain et al., $2014{ }^{(10)}$ & Low risk & NR & High risk & Moderate risk & NR & Low risk \\
\hline Galusca et al., $2015^{(23)}$ & Low risk & NR & High risk & Low risk & NR & Low risk \\
\hline Germain et al., $2016^{(27)}$ & Low risk & NR & High risk & Low risk & NR & Low risk \\
\hline Gunes et al., $2016^{(47)}$ & Low risk & NR & High risk & Low risk & NR & Low risk \\
\hline Ling et al., $2016^{(31)}$ & Low risk & NR & High risk & Moderate risk & NA & NA \\
\hline Estour et al., $2017^{(8)}$ & Low risk & NR & High risk & Low risk & NR & Low risk \\
\hline Galusca et al., $2018^{(19)}$ & Low risk & NR & High risk & Low risk & NR & Low risk \\
\hline Florent et al, 2019 & Low risk & NR & High risk & Moderate risk & Moderate risk & Low risk \\
\hline Margaritelis et al., $2019^{(40)}$ & Low risk & NR & High risk & Moderate risk & NR & Low risk \\
\hline Marra et al., $2019^{(18)}$ & Moderate risk & NR & High risk & Low risk & NR & Low risk \\
\hline Riveros-McKay et al., $2019^{(30)}$ & Low risk & NR & High risk & Low risk & Low risk & Low risk \\
\hline
\end{tabular}




\section{Discussion}

The literature shows a growing number of clinical trials enrolling underweight participants without apparent disorders in their energy balance, suggesting a constitutional origin of thinness. These studies, however, reveal a high heterogeneity when it comes to the employed definition and diagnosis of CT; as well as a high diversity in the fields of study. In that context, we proposed here a systematic analysis of the clinical trials that enrolled participants with $\mathrm{CT}$ in order to propose a better definition and diagnosis of CT.

\section{The need for a clear terminology}

The lack of consensus and visibility concerning CT is probably due to the lack of common terminology. Among the 35 studies considered in the present systematic review, $7^{(16,24,34,39,40,44,45)}$ did not used the key terms "constitutional thinness" or "constitutional leanness". This makes highly probable that people might not detect those references while conducting simple scientific or systematic researches. For example, Farooqi and her research team who conducted a very interesting genetic research on CT ${ }^{(30)}$ preferentially used the "persistent/healthy thinness" expression even if "constitutional thinness" is still found once ${ }^{(30)}$. In addition, studies enrolling "lean" or "underweight" groups need to be particularly screened. Most of the time, the "lean" term refers to normal-weight individuals and "underweight" term to undernourishment, but confusingly, these terms also remain found in the literature to designate CT individuals. Thus, we would privilege a common terminology, such as "constitutional thinness" or "constitutional leanness" designations. Since CT individuals do not seem to be characterized by a very low body fat percentage despite their low BMI ${ }^{(8,10,11,17,19,23-27)}$, we would favor the terminology of "constitutional thinness" which therefore seems more appropriate than "constitutional leanness". A common terminology would drastically facilitate the referencing of CT in research databases and increase its visibility. 


\section{Thinness threshold}

As underlined in different studies, dealing with thinness first requires to properly set a threshold for this thinness ${ }^{8,15,57)}$. The World Health Organization (WHO) defines different thresholds, based on BMI cutoffs: grade 1 - mild thinness $\left(17.00-18.49 \mathrm{~kg} \cdot \mathrm{m}^{-2}\right)$, grade 2 - moderate thinness $\left(16.00-16.99 \mathrm{~kg} \cdot \mathrm{m}^{-2}\right)$, and grade 3 - severe thinness $\left(<16.00 \mathrm{~kg} \cdot \mathrm{m}^{-2}\right){ }^{(57,58)}$. Thus, the WHO uses the BMI measurement to provide demarcation points. Of the 35 included studies, $22^{(7-10,16,19,21-28,30,31,35,37,40,41,45,47)}$ also used BMI cut-offs and one study ${ }^{(44)}$ used a threshold of BMI percentile ( $\leq 15^{\text {th }}$ BMI percentile). BMI cut-offs ranged from $16.5^{(7,9,21-23,28)}$ to $20.0^{(35,40)} \mathrm{kg} . \mathrm{m}^{-2}$ for studies using a BMI threshold and mean BMI ranged from $15.7^{(9,11)}$ to $22.5^{(42)}$ in individuals with CT; revealing a high heterogeneity in BMI values. Two studies ${ }^{(40,43)}$ used percentages of FM to define a thinness cut-off. From an etymological point of view, "leanness" defines a low body fat content and interestingly, Maffetone et al. proposed the use of the "underfat" term instead of "underweight" (59). Nevertheless, Maffetone et al. proposed this terminology considering thinness due to chronic illness or eating disorders, not thinness due to a constitutional origin ${ }^{(59)}$. Despite their low BMI, CT individuals have been suggested to present a non-blunted FM percentage (7,8,10,11,17,19,23-27), unlike AN individuals whose FM seems significantly lower compared to CT people $(7,8,11,17,23,24)$. The use of a body fat percentage threshold does not seem yet adequate to diagnose CT and could on the contrary lead to misdiagnosis. While we therefore suggest that "underfat" might not be an appropriate term in the context of CT, further studies using similar inclusion criteria and methodologies are required to provide more evidence about body composition in CT. Two studies ${ }^{(33,34)}$ focused their definition of thinness on a percentage of ideal bodyweight and two studies ${ }^{(36,42)}$ argued that silhouette ratings were a better choice to base their definition of thinness. Nevertheless, silhouette ratings led to the inclusion of individuals with a relatively high BMI of $20.3 \mathrm{~kg} \cdot \mathrm{m}^{-2}(36)$ in females and $22.5 \mathrm{~kg} . \mathrm{m}^{-2}(42)$ in males; whose CT diagnosis was therefore highly debatable. Seven studies $(11,17,18,20,38,39,46)$ did not clearly report any threshold for their definition of thinness. Thus, the systematic review revealed that studies do not systematically point out a cut-off to define thinness. In addition, large variability in both the used criteria and cut-off values was observed. In that context, it seems complex to propose specific 
recommendations concerning a thinness threshold. However, given the BMI cut-offs of the WHO ${ }^{(57,58)}$, we would recommend not to enroll CT individuals with a BMI exceeding $18.49 \mathrm{~kg} . \mathrm{m}^{-2}$.

\section{Weight history}

Weight fluctuation and duration of fluctuations are other important parameters that should accompany consideration of the thinness degree. The present systematic review showed that weight history was well taken into consideration with 25 studies ${ }^{(7-10,16-19,21-23,27,28,30,31,35,36,38-40,42,44-47)}$ reporting this criterion. However, there was a high heterogeneity in modalities: 4 studies ${ }^{(16,39,40,45)}$ reported a stable weight for a certain period of time before the experiment (ranging from one week ${ }^{(45)}$ to 2 years ${ }^{(16,39,40)}$ ) and 21 studies ${ }^{(7-10,17-19,21-23,27,28,30,31,35,36,38,42,44,46,47)}$ reported it for a longer period throughout the growth period and/or the post-pubertal period. In 1982, Apfelbaum and Sachet already stressed the need to consider the weight history of CT patients and to differentiate between slimness and slimming ${ }^{(15)}$. Indeed, weight history opposes $\mathrm{CT}$ from $\mathrm{AN}{ }^{(5,7,8)}$. Contrary to $\mathrm{AN}$ that is characterized by a curve break at the onset of anorexic tendencies, the diagnosis of CT should be supported by a low BMI (approximately the $3^{\text {rd }}$ percentile) during all the growth period and by a stable bodyweight throughout the post-pubertal period ${ }^{(5,7,8)}$. In addition, CT seems to be a heritable trait ${ }^{(29,30)}$, leading to CT families ${ }^{(7)}$. For three generations, an average of 2.5 thin subjects per family is found CT for only 0.5 per family in AN ${ }^{(7)}$. Thus, the presence of other thin individuals in familial history can also reinforce a CT diagnosis.

\section{Absence of eating disorders, associated pathology, and over-exercising}

Potential eating disorders and associated diseases, as well as an energy imbalance caused by a high energy expenditure through physical activity, need to be taken into account to properly identify CT $(5,8,10,19,21,28,31)$. In the 35 included papers, the absence of eating disorders was well-considered: only 3

papers ${ }^{(39,40,47)}$ did not consider this criterion. Although well-considered, this absence of eating disorders is most of the time simply mentioned or implicitly suggested without any details regarding its assessment. Only $26 \%(10,30,31,35,36,41,42,44,46)$ of the included studies used specific tools, like questionnaires 
or interviews, to confirm the absence of eating disorders and only 2 studies ${ }^{(10,31)}$ associated questionnaires with the assessment of the following nutritional biomarkers: normal IGF-1, estradiol, $\mathrm{FT}_{3}$, mean cortisol, and non-blunted leptin. In addition, questionnaires and interviews used were highly heterogeneous, using different versions, rarely reporting thresholds, and if so, with different thresholds. This observation shows the real need to adopt harmonized and common methods to robustly detect eating disorders. Concerning the absence of associated pathology, this criterion was well-considered in the selected papers: only 7 studies ${ }^{(11,24,35,36,38,42,45)}$ did not report it. Since some studies may have taken into account some diagnostic parameters without explicitly detailing them in their inclusion process, we assume that some diagnostic parameters may have been slightly underestimated. Regarding physical activity, $63 \%{ }^{(8,9,11,18-21,24,26,27,33-38,41-46)}$ of the included studies did not report any physical activity level in their inclusion criteria. Ten articles $(7,16,17,22,25,28,30,39,40,47)$ simply mentioned the absence of high physical activity level and only 3 articles ${ }^{(10,23,31)}$ actually assessed physical activity level, using the MOSPA questionnaire ${ }^{(56)}$. Importantly, the relevance of the MOSPA questionnaire ${ }^{(56)}$ should be discussed. This questionnaire has been validated ${ }^{(56)}$ among 50 pregnant women only, and several limitations in the methodological approaches of its validation need to be recognized ${ }^{(56)}$. The thresholds used to define the different physical activity levels differ: 2 articles ${ }^{(39,40)}$ specified that participants did not spend more than one hour per week in sport activities, one article ${ }^{(31)}$ considered the practice of more than 3 sessions of physical activity per week as an exclusion criterion and one article ${ }^{(30)}$ excluded all participants who stated that they exercised more than 3 times a week or with an intensity exceeding 6 METs for any duration or frequency ${ }^{(55)}$. Altogether, these observations raised a real need to precisely describe the population in terms of type, duration, frequency, and intensity of physical activity with validated questionnaires but also with a more objective method such as accelerometry. Interestingly, spontaneous repeated muscle contractions in daily life, like fidgeting, were also suggested to be a relevant parameter to evaluate in CT for future studies ${ }^{(10,17,20,31)}$. 


\section{Weight gain resistance/desire}

Of the included articles, less than half of them ${ }^{(7-10,16,21-23,27,28,31,43,46,47)}$ mentioned weight gain resistance/desire in their inclusion criteria of CT people, and most of these articles have been written by members of the same research team ${ }^{(7-10,21-23,27,28,31)}$. Of the $14 \operatorname{articles}^{(7-10,16,21-23,27,28,31,43,46,47)}$ mentioning this weight gain resistance/desire, $12^{(7-10,21-23,27,28,31,46,47)}$ used the idea of a "desire" to gain weight, one study (16) mentioned a complaint about being chronically underweight, another study ${ }^{(43)}$ reported difficulty in gaining weight, and no studies used the term "resistance" to weight gain. Even if the desire to gain weight is actually, most of the time, the main reason for medical consultation in CT ${ }^{(5)}$ and definitely differentiates $\mathrm{CT}$ from AN, we suggest here that an individual with $\mathrm{CT}$ might not present a strong desire to gain weight despite a physiological weight gain resistance, to the same extent that obesity is not defined as the subject's "willingness" to lose weight. In the case of CT, it may seem more accurate to define it as a "resistance" to gain weight; that can result in a desire to gain weight - but not necessarily. Indeed, CT was found to be the first human model of physiological weight gain resistance ${ }^{(10)}$ and several publications proposed supplements and treatments to help CT people gain weight, a few decades earlier ${ }^{(3,4,15,60)}$ or more recently ${ }^{(10,31)}$. Bulik and Allison even proposed the following definition of CT: "constitutional protection against the need to diet in order to maintain a low body weight" (29).

\section{Female gender predominance and amenorrhea}

A female gender predominance was observed with 26 studies ${ }^{(7-11,16,17,19-28,33-41)}$ that were conducted among females exclusively, 6 studies ${ }^{(30,31,44-47)}$ on both genders and 3 studies ${ }^{(18,42,43)}$ in males exclusively. As the systematic review was performed on clinical studies, it seems to us that this observation probably only illustrates the lower consultation rate in men and we encourage further researches in both genders; as CT is not a sex-specific condition. The presence of menses in the diagnosis of CT was widely taken into account: only 6 studies ${ }^{(19,30,33,36,44,45)}$ did not mention this criterion of the 32 studies ${ }^{(7-11,16,17,19-28,30,31,33-41,44-47)}$ enrolling females. Although the absence of amenorrhea was wellconsidered in the studies, the removal of this criterion from the revised DSM-V ${ }^{(13)}$ can lead to new 
difficulties in the differential diagnosis between $\mathrm{AN}$ and $\mathrm{CT}^{\left({ }^{(8)}\right.}$. It seems however relevant to us to verify the absence of amenorrhea in the diagnosis of CT.

\section{Recommendations in the diagnosis of CT}

The systematic review of clinical trials that enrolled participants with CT definitely revealed the real need to adopt both a common terminology and a well-defined diagnosis of CT. Based on the present results, we collectively propose here the key term "constitutional thinness" to be used. Using the "constitution" term to refer to the innate and natural cause of thinness seems of particular interest since it also helps clarify the distinction with other behavioral or pathological origins of thinness. In this respect, it seems essential to systematically exclude energy imbalance caused by inappropriately low energy intake (eating disorders) and/or an inappropriately high exercise-induced energy expenditure, using validated tools. Ideally, eating behavior should be evaluated not only with common validated questionnaires or interviews using specific thresholds, but also with the assessment of nutritional biomarkers. If possible, the absence of over-exercising should not only be declarative but also measured with robustly validated questionnaires or even by accelerometry technique. Although amenorrhea has been removed from the definition of AN in the DSM-V ${ }^{(13)}$, it seems relevant to consider the presence of physiological menstruations in the diagnosis of CT. In addition, weight gain resistance and weight history also need to be taken into consideration in the diagnosis. Finally, the question of defining a strict threshold for thinness remains complex and arbitrary. Even though BMI assessment is associated with various limitations ${ }^{(59)}$, we would tend to favor this measurement as long as it is very common and simple to perform. Conversely, we recommend not to use the percentage of body fat as a maximal threshold since CT does not seem to be characterized by a low body fat percentage ${ }^{(8,10,11,17,19,23-27)}$. Given the BMI cut-offs of the $\mathrm{WHO}{ }^{(57,58)}$, we propose that $\mathrm{CT}$ should not be discussed with a BMI exceeding the value of $18.49 \mathrm{~kg} \cdot \mathrm{m}^{-2}$. Beyond these essential criteria for CT diagnosis, some studies seem to suggest certain common characteristics in CT groups. In comparison to people with AN, CT individuals might display higher REE and REE-to-FFM ${ }^{(7,17,18)}$ (although it does not seem significant in two studies ${ }^{(8,16)}$ ), nonblunted FM percentages despite their low BMI ${ }^{(7,8,11,17,23,24)}$, and different profiles of appetite-regulating 
hormones $\left.{ }^{(9,11,21-23)}\right)$. If these types of results were supported by a substantial number of studies and clinical evidence, they could be used as new criteria for the distinction of CT from AN in the future, which remains to be robustly demonstrated. In order to visually synthesize the potential actual recommendations in CT diagnosis, based on this systematic analysis, a decision tree is proposed in Figure 2.

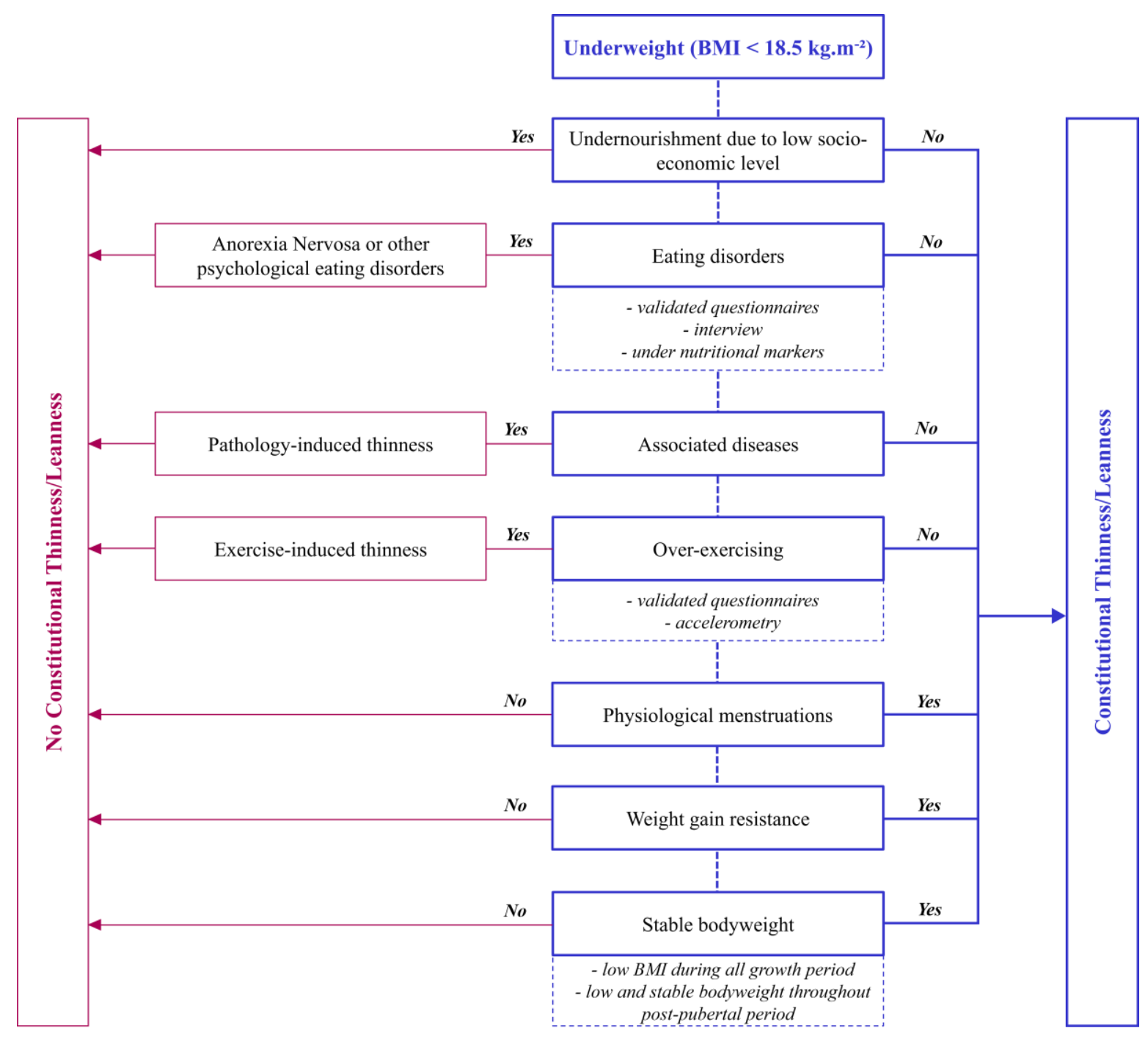

Figure 2. Decision tree in the diagnosis of constitutional thinness

On top of the inclusion criteria used by the selected studies, their methodologies must also be considered when interpreting our results as analyzed and presented in our Risks of Bias table (Table 2). Indeed, as reported in Table 2, 34 out of the 35 included studies present a high risk for the "blinding of participants 
and personnel", which might affect the obtained results when it comes for instance to the evaluation of energy intake, eating profiles or physical activity that could be affected by the non-blinding of participants or personnel. This interpretation of our analyses must also consider the high proportion of studies presenting a moderate-to-high risk regarding the attrition bias, or even unreported data.

\section{Conclusion}

The present review used a systematic approach to identify any clinical trials that enrolled individuals with CT, particularly focusing on the methods used to define and diagnose CT. The employed methodology led us to identify 35 clinical trials enrolling a group of participants with CT. This clearly pointed out a relatively reduced number of studies interested in this condition. In addition, the definition and the diagnostic features of CT were found highly heterogeneous in these studies. Terminology and thinness thresholds do not reach consensus and a high heterogeneity was also observed regarding the assessment of weight history, weight gain resistance and the presence of physiological menses. The absence of eating disorders, associated pathology or over-exercising was not systematically verified and if so, with various methodological approaches. This systematic review points out the essential need not only to be aware of the existence of CT but also to harmonize our medical and scientific practices in the definition and diagnosis of CT. Altogether, the present results led us to propose a decision tree that could help practitioners and researchers better define and diagnose constitutional thinness, in a potentially more harmonized way. Importantly, not only the proposed decision tree has been elaborated based on clinically relevant indicators that have to be considered for the diagnosis of CT, but it also proposes different evaluation alternatives (from self-reported eating questionnaires to undernutrition physiological makers for instance); guaranteeing its clinical feasibility and applicability. 
Acknowledgements: The authors are thankful to the documentalists of the Health Library of Clermont Auvergne University for their help in the article search process.

Financial support: This research received no specific grant from any funding agency, commercial or not-for-profit sectors.

Conflict of Interest: None.

Author contributions: Conceptualization, piloting of the study selection process, and formal screening of search results against eligibility criteria: MB, JV, DT; Data extraction and analysis: MB; Writing the article: MB; Supervision and revision of the article: JV, DT, NG, BG, DC. 


\section{References}

1. Grafe E (1933) Metabolic diseases and their treatment - Chapter VIII: Constitutional undernutrition of doubtful origin. Lea \& Febiger.

2. Passmore R, Meiklejohn AP, Dewar AD, et al. (1955) Energy utilization in overfed thin young men. Br. J. Nutr. 9, 20-27.

3. Genest J, Adamkiewicz L, Robillard R, et al. (1955) Clinical uses of rauwolfia. II. In psoriasis and in constitutional leanness. Can. Med. Assoc. J. 72, 490-491.

4. Wissmer B (1953) [Treatment of constitutional thinness]. Arch. Mal. Appar. Dig. Mal. Nutr. 42, 149-157.

5. Estour B, Galusca B \& Germain N (2014) Constitutional thinness and anorexia nervosa: a possible misdiagnosis? Front. Endocrinol. 5, 175.

6. World Health Organization Prevalence of underweight among adults. https://www.who.int/data/gho/data/indicators/indicator-details/GHO/prevalence-of-underweightamong-adults-bmi-18-(crude-estimate)-(-) (accessed March 2020).

7. Bossu C, Galusca B, Normand S, et al. (2007) Energy expenditure adjusted for body composition differentiates constitutional thinness from both normal subjects and anorexia nervosa. Am. J. Physiol. Endocrinol. Metab. 292, E132-137.

8. Estour B, Marouani N, Sigaud T, et al. (2017) Differentiating constitutional thinness from anorexia nervosa in DSM 5 era. Psychoneuroendocrinology 84, 94-100.

9. Germain N, Galusca B, Le Roux CW, et al. (2007) Constitutional thinness and lean anorexia nervosa display opposite concentrations of peptide YY, glucagon-like peptide 1, ghrelin, and leptin. Am. J. Clin. Nutr. 85, 967-971.

10. Germain N, Galusca B, Caron-Dorval D, et al. (2014) Specific appetite, energetic and metabolomics responses to fat overfeeding in resistant-to-bodyweight-gain constitutional thinness. Nutr. Diabetes 4, e126.

11. Tolle V, Kadem M, Bluet-Pajot M-T, et al. (2003) Balance in ghrelin and leptin plasma levels in anorexia nervosa patients and constitutionally thin women. J. Clin. Endocrinol. Metab. 88, 109-116. 12. Guy-Grand B \& Basdevant A (1982) [Etiopathogenesis and general orientation in the diagnosis of thinness]. Rev. Prat. 32, 237-243.

13. American Psychiatric Association (2013) Diagnostic and Statistical Manual of Mental Disorders: DSM-5, 5th ed., Washington, D.C. .

14. Stewart M-C, Keel PK \& Schiavo RS (2006) Stigmatization of anorexia nervosa. Int. J. Eat. Disord. 39, 320-325.

15. Apfelbaum M \& Sachet P (1982) [Constitutional thinness]. Rev. Prat. 32, 245-247.

16. Scalfi L, Coltorti A, Borrelli R, et al. (1992) Postprandial thermogenesis in leanness and anorexia nervosa. Ann. Nutr. Metab. 36, 48-54.

17. Marra M, Pasanisi F, Montagnese C, et al. (2007) BMR variability in women of different weight. Clin. Nutr. Edinb. Scotl. 26, 567-572.

18. Marra M, Sammarco R, De Filippo E, et al. (2019) Resting energy expenditure, body composition and phase angle in anorectic, ballet dancers and constitutionally lean males. Nutrients 11, 502.

19. Galusca B, Verney J, Meugnier E, et al. (2018) Reduced fibre size, capillary supply and mitochondrial activity in constitutional thinness' skeletal muscle. Acta Physiol. Oxf. Engl. 224, e13097. 20. Pasanisi F, Pace L, Fonti R, et al. (2013) Evidence of brown fat activity in constitutional leanness. J. Clin. Endocrinol. Metab. 98, 1214-1218.

21. Germain N, Galusca B, Grouselle D, et al. (2009) Ghrelin/obestatin ratio in two populations with low bodyweight: constitutional thinness and anorexia nervosa. Psychoneuroendocrinology 34, 413-419.

22. Galusca B, Jeandel L, Germain N, et al. (2012) Orexigenic neuropeptide 26RFa: new evidence for an adaptive profile of appetite regulation in anorexia nervosa. J. Clin. Endocrinol. Metab. 97, 20122018. 
23. Galusca B, Prévost G, Germain N, et al. (2015) Neuropeptide Y and $\alpha$-MSH circadian levels in two populations with low body weight: anorexia nervosa and constitutional thinness. PloS One 10, e0122040.

24. Fernández-García D, Rodríguez M, García Alemán J, et al. (2009) Thin healthy women have a similar low bone mass to women with anorexia nervosa. Br. J. Nutr. 102, 709-714.

25. Marra M, Caldara A, Montagnese C, et al. (2009) Bioelectrical impedance phase angle in constitutionally lean females, ballet dancers and patients with anorexia nervosa. Eur. J. Clin. Nutr. 63, 905-908.

26. Hasegawa A, Usui C, Kawano H, et al. (2011) Characteristics of body composition and resting energy expenditure in lean young women. J. Nutr. Sci. Vitaminol. (Tokyo) 57, 74-79.

27. Germain N, Viltart O, Loyens A, et al. (2016) Interleukin-7 plasma levels in human differentiate anorexia nervosa, constitutional thinness and healthy obesity. PloS One 11, e0161890.

28. Galusca B, Zouch M, Germain N, et al. (2008) Constitutional thinness: unusual human phenotype of low bone quality. J. Clin. Endocrinol. Metab. 93, 110-117.

29. Bulik CM \& Allison DB (2001) The genetic epidemiology of thinness. Obes. Rev. Off. J. Int. Assoc. Study Obes. 2, 107-115.

30. Riveros-McKay F, Mistry V, Bounds R, et al. (2019) Genetic architecture of human thinness compared to severe obesity. PLoS Genet. 15, e1007603.

31. Ling Y, Galusca B, Hager J, et al. (2016) Rational and design of an overfeeding protocol in constitutional thinness: Understanding the physiology, metabolism and genetic background of resistance to weight gain. Ann. Endocrinol. 77, 563-569.

32. Higgins JPT \& Green S (2008) Cochrane handbook for systematic reviews of interventions. Wiley Online Libr., 187-235.

33. Schneider L, Heard I, Bréart G, et al. (1979) [Maternal thinness and pregnancy]. Arch. Fr. Pediatr. 36, 1068-1074.

34. van Binsbergen CJ, Coelingh Bennink HJ, Odink J, et al. (1990) A comparative and longitudinal study on endocrine changes related to ovarian function in patients with anorexia nervosa. J. Clin. Endocrinol. Metab. 71, 705-711.

35. Petretta M, Bonaduce D, Scalfi L, et al. (1997) Heart rate variability as a measure of autonomic nervous system function in anorexia nervosa. Clin. Cardiol. 20, 219-224.

36. Slof R, Mazzeo S \& Bulik CM (2003) Characteristics of women with persistent thinness. Obes. Res. 11, 971-977.

37. Tagami T, Satoh N, Usui T, et al. (2004) Adiponectin in anorexia nervosa and bulimia nervosa. J. Clin. Endocrinol. Metab. 89, 1833-1837.

38. Miljic D, Pekic S, Djurovic M, et al. (2006) Ghrelin has partial or no effect on appetite, growth hormone, prolactin, and cortisol release in patients with anorexia nervosa. J. Clin. Endocrinol. Metab. 91, 1491-1495.

39. Paschalis V, Nikolaidis MG, Theodorou AA, et al. (2013) The effects of eccentric exercise on muscle function and proprioception of individuals being overweight and underweight. J. Strength Cond. Res. 27, 2542-2551.

40. Margaritelis NV, Theodorou AA, Kyparos A, et al. (2019) Effect of body composition on redox homeostasis at rest and in response to exercise: the case of underfat women. J. Sports Sci. 37, 1-8.

41. Florent V, Baroncini M, Jissendi-Tchofo P, et al. (2019) Hypothalamic structural and functional imbalances in anorexia nervosa. Neuroendocrinology.

42. Mazzeo SE, Slof RM, Tozzi F, et al. (2004) Characteristics of men with persistent thinness. Obes. Res. 12, 1367-1369.

43. Diaz EO, Prentice AM, Goldberg GR, et al. (1992) Metabolic response to experimental overfeeding in lean and overweight healthy volunteers. Am. J. Clin. Nutr. 56, 641-655.

44. Hinney A, Barth N, Ziegler A, et al. (1997) Serotonin transporter gene-linked polymorphic region: allele distributions in relationship to body weight and in anorexia nervosa. Life Sci. 61, PL 295303.

45. Bosy-Westphal A, Reinecke U, Schlörke T, et al. (2004) Effect of organ and tissue masses on resting energy expenditure in underweight, normal weight and obese adults. Int. J. Obes. Relat. Metab. Disord. J. Int. Assoc. Study Obes. 28, 72-79. 
46. Santonicola A, Siniscalchi M, Capone P, et al. (2012) Prevalence of functional dyspepsia and its subgroups in patients with eating disorders. World J. Gastroenterol. 18, 4379-4385.

47. Gunes A, Yildırım Bas F, Arslan B, et al. (2016) Assessment of corneal parameters in patients with constitutional thinness using scheimpflug imaging. Semin. Ophthalmol. 33, 175-178.

48. Stunkard AJ \& Messick S (1985) The three-factor eating questionnaire to measure dietary restraint, disinhibition and hunger. J. Psychosom. Res. 29, 71-83.

49. van Strien T, Frijters JER, Bergers GPA, et al. (1986) The Dutch Eating Behavior Questionnaire (DEBQ) for assessment of restrained, emotional, and external eating behavior. Int. J. Eat. Disord. 5, 295-315.

50. Cooper Z \& Fairburn C (1987) The eating disorder examination: a semi-structured interview for the assessment of the specific psychopathology of eating disorders. Int. J. Eat. Disord. 6, 1-8.

51. Garner DM, Olmstead MP \& Polivy J (1983) Development and validation of a multidimensional eating disorder inventory for anorexia nervosa and bulimia. Int. J. Eat. Disord. 2, 15-34.

52. Cooper PJ, Taylor MJ, Cooper Z, et al. (1987) The development and validation of the body shape questionnaire. Int. J. Eat. Disord. 6, 485-494.

53. Morgan JF, Reid F \& Lacey JH (1999) The SCOFF questionnaire: assessment of a new screening tool for eating disorders. BMJ 319, 1467-1468.

54. World Health Organization (1990) Composite International Diagnostic Interview (CIDI): a) CIDI-interview version 1.0, b) CIDI-user manual, c) CIDI-training manual, d) CIDI-computer programs. .

55. World Health Organization What is moderate-intensity and vigorous-intensity physical activity? WHO. https://www.who.int/dietphysicalactivity/physical_activity_intensity/en/(accessed July 2019).

56. Iqbal R, Rafique G, Badruddin S, et al. (2006) Validating MOSPA questionnaire for measuring physical activity in Pakistani women. Nutr. J. 5, 18.

57. World Health Organization Expert Committee (1995) Physical status: the use and interpretation of anthropometry. 423.

58. World Health Organization (2019) Body mass index - BMI. http://www.euro.who.int/en/healthtopics/disease-prevention/nutrition/a-healthy-lifestyle/body-mass-index-bmi (accessed July 2019).

59. Maffetone PB, Rivera-Dominguez I \& Laursen PB (2017) Overfat and underfat: new terms and definitions long overdue. Front. Public Health 4, 279.

60. Aubertin E (1953) [Treatment of constitutional thinness]. J. Med. Bord. 130, 676-681. 